\title{
Robust Econometric Inference with Mixed Integrated and Mildly Explosive Regressors
}

\author{
Peter C.B. Phillips* Ji Hyung Lee ${ }^{\dagger}$
}

November, 2015

\begin{abstract}
This paper explores in several prototypical models a convenient inference procedure for nonstationary variable regression that enables robust chi-square testing for a wide class of persistent and endogenous regressors. The approach uses the mechanism of self-generated instruments called IVX instrumentation developed by Magdalinos and Phillips (2009b). We first show that these methods remain valid for regressors with local unit roots in the explosive direction and mildly explosive roots, where the roots are further from unity in the explosive direction than $O\left(n^{-1}\right)$. It is also shown that Wald testing procedures remain robust for multivariate regressors with certain forms of mixed degrees of persistence. These robustifications are useful in econometric inference, for example, when there are periods of mildly explosive trends in some or all of time series employed in the analysis but the exact knowledge on the regressor persistence is unavailable. Some aspects of the choice of the IVX instruments are investigated and practical guidance is provided but the issue of optimal IVX instrument choice remains unresolved. The methods are straightforward to apply in practical work such as predictive regression applications in finance.

Keywords: Chi-square, Instrumentation, IVX methods, Local to unity, Mild integration, Mild explosiveness, Predictive regression, Robustness.
\end{abstract}

JEL classification: $\mathrm{C} 22$

\section{Introduction}

Many economic and financial time series exhibit characteristics that include temporary periods of explosive behavior. For macroeconomic series Stock (1991, Table 2) showed that $90 \%$ confidence intervals for the autoregressive (AR) roots of the Nelson-Plosser data set contain explosive parameter regions in all but one series (the unemployment rate). For financial series Campbell and Yogo (2006, Table 4) found that 95\% confidence intervals for the AR coefficient of the S\&P 500

${ }^{*}$ Yale University, University of Auckland, Singapore Management University \& University of Southampton. Email: peter.phillips@yale.edu

${ }^{\dagger}$ University of Illinois. Email: jihyung@illinois.edu 
dividend-price ratio and other series over long historical periods do not rule out explosive roots. In addition to these empirical findings, periodically occurring booms and episodes of financial exuberance support at least temporary explosive trends in economic and financial data. The idea that there are subperiods of explosive roots in economic and financial series is formally analyzed and empirically confirmed in several recent papers (Phillips et al., 2011, 2015a, 2015b; Phillips and Yu, 2011).

There has been growing interest in predictive regressions of the type used in the study by Campbell and Yogo. When the regressors in such models display some degree of persistence, inference procedures need to be robust to the value of the persistence parameter to ensure validity even asymptotically. The robustness requirements become more demanding in cases where there are many regressors with possibly different degrees of persistence. To address some of these complexities in inference in regressions with persistence regressors, Magdalinos and Phillips (2009b, hereafter MP) recently introduced a novel IV procedure (called IVX regression) that provides robust chisquare inference in a much wider vicinity of unity than existing studies that have typically only considered the near integrated (local to unity) single regressor case. In particular, MP showed that self-normalized IVX test statistics have an asymptotically pivotal chi-square distribution for multivariate regressors that may be integrated, near integrated, or mildly integrated and which thereby fall within a vector autoregressive framework (Lutkepohl, 2005) while allowing for more general time series inputs than martingale differences. The tests have been successfully used in applied work on predictive regressions (Kostakis et al., 2014; Gonzalo and Pitarakis, 2012). IVX methods have been also studied in long-horizon regression applications (Phillips and Lee, 2013) and in quantile regression (Lee, 2014) contexts.

The present paper is an exploratory study in protypical cases that show how the IVX method-

ology extends to a wider range of potential regressors that includes local unit root (LUR) explosive and mildly explosive roots, thereby covering periods of exuberance in economic and financial data. The limit theory involves some novel developments in the mildly explosive case, wherein the latent IVX instrument which depends on the true values of the localizing coefficients may no longer dominate the asymptotics. The chi-square limit theory of the same self-normalized test statistics of MP is shown to continue to be valid in this wider setting. We also confirm that the limit theory is robust under mixed degrees of persistence, allowing for the simultaneous presence of local to unity (or mildly integrated) roots and mildly explosive roots. These extensions show that IVX regression provides a framework of test procedures that can cover a large class of persistent regressors whose individual characteristics may differ from each other. While the results in the present paper show validity in a prototypical system, the wider practical implication is that empirical researchers may use this framework without having to be specific about the particular properties of individual regressors. Further research that is presently underway aims to provide a unified treatment that justifies the use of this approach for a comparably wide class of regressors in a general multivariate setting.

The paper is organized as follows. Section 2 develops the limit theory for IVX regression under 
locally and mildly explosive roots and demonstrates robustness. Section 3 extends these robustness results to cases where the regressors have mixed degrees of persistence or explosive behavior. Section 4 discusses issues associated with the choice of the IVX tuning parameter and provides simulation results. Section 5 concludes. Some technical derivations, supporting lemmas, and proofs of the main results in the paper are contained in the Appendix. An online supplement (Phillips and Lee, 2015c) provides an empirical illustration as well as further lemmas and proofs that are useful in establishing the results of the paper.

\section{IVX Regression with Explosive Roots}

In what follows, we use the spectral norm $\|M\|=\max _{i}\left\{\lambda_{i}^{1 / 2}: \lambda_{i}=\right.$ an eigenvalue of $\left.M^{\prime} M\right\}$. Other norms, such as the $L_{1}$ and $L_{2}$ norms, are specified in what follows as needed using the notation $\|\cdot\|_{L_{i}}(i=1,2)$.

\subsection{Framework}

We use the framework of MP for the following system:

$$
\begin{aligned}
y_{t} & =A x_{t}+u_{0 t}, \\
x_{t} & =R_{n} x_{t-1}+u_{x t}, \\
R_{n} & =I_{K}+\frac{C}{n^{\alpha}}, \text { for some } \alpha>0,
\end{aligned}
$$

where $A$ is an $m \times K$ coefficient matrix and $C=\operatorname{diag}\left(c_{1}, c_{2}, \ldots, c_{K}\right)$ represents the localizing coefficients in the multivariate regressors. Use of a diagonal matrix $C$ in (2.1) has become standard practice in the literature because it helps to distinguish individual components of $x_{t}$ in terms of their integration properties 1

The IVX approach of MP allowed the regressors $x_{t}$ to be (I1) integrated $(C=0)$, (I2) near integrated $(C<0, \alpha=1)$ and (I3) mildly integrated $(C<0, \alpha \in(0,1))$, and developed an inference procedure that is robust to the precise degree of integration 2 . We will show these results are robust under the same framework but with (I4) local to unity on the explosive side of unity $(C>0, \alpha=1)$ and (I5) mildly explosive roots $(C>0, \alpha \in(0,1))$, as well as possibly mixed versions (I6) of these roots.

As in MP or Kostakis et al. (2014), the initial conditions for the driver regressor $x_{t}$ in (I1)-(I2) and (I3) could be $x_{0}=o_{p}\left(n^{1 / 2}\right)$ or $x_{0}=o_{p}\left(n^{\alpha / 2}\right)$, respectively. Indeed, $x_{0}=o_{p}\left(n^{\alpha / 2}\right)$ also applies in the (I4) case. For (I5), in view of Lemma 2.1 and 2.2, we could impose $x_{0}=o_{p}\left(n^{\alpha / 2}\left\|R_{n}\right\|^{n}\right)=$

\footnotetext{
${ }^{1}$ The general case of matrix $C$ was considered in the original work on near integrated vector processes (Phillips, 1988) with several examples that illustrate the impact of off diagonal elements on the limit theory. These typically introduce additional components in the limit that take the form of smoothed diffusions.

${ }^{2}$ Some other cases of neglected nonlinearities (such as structural breaks) and linkages to unit root or explosive behavior may be included in the set up. For instance, Diebold and Inoue (2001) have shown that stochastic regime switching is easily confused empirically with long memory; and certain nonlinear functions of $I(1)$ processes are well-known to behave like stationary long memory processes (Miller and Park, 2010; Kasparis et al., 2014).
} 
$o_{p}\left(n^{\alpha / 2} e^{\|C\| n^{1-\alpha}}\right)$. Other possibilities, where the initial conditions have larger orders may also be analyzed and, as in Phillips and Magdalinos (2009) such cases typically impact the asymptotic theory.

The system (2.1) is prototypical and may readily be extended to cases that suit particular empirical applications. For example, intercepts and deterministic regressors are commonly present in practice in predictive systems like (2.1). Also, the generating mechanism for the predictors $x_{t}$ may also involve deterministic components or trianguilar system versions of such components that upon standardization lead to functional (or other) stochastic process limits with comparable deterministic components (e.g. Phillips, Shi and Yu, 2014). Theory and implementation of the methods discussed here under some extensions of this type are considered later in the paper.

For the time series structure of the innovations that appear in 2.10 it is convenient to maintain one of the following two assumptions, which allow for weak dependence and conditional heterogeneity.

\section{Assumption 2.1 (LP)}

$$
\begin{gathered}
u_{t}:=\left[\begin{array}{l}
u_{0 t} \\
u_{x t}
\end{array}\right]=\sum_{j=0}^{\infty} F_{j} \varepsilon_{t-j}, \quad \varepsilon_{t} \sim i i d(0, \Sigma), \Sigma>0, E\left\|\varepsilon_{1}\right\|^{4}<\infty, \\
F_{0}=I_{m+K}, \quad \sum_{j=0}^{\infty} j\left\|F_{j}\right\|<\infty, F(z)=\sum_{j=0}^{\infty} F_{j} z^{j} \text { and } F(1)=\sum_{j=0}^{\infty} F_{j}>0 .
\end{gathered}
$$

\section{Assumption $2.2(\mathrm{NLP})$}

$$
u_{t}=g\left(\xi_{t}\right)
$$

where the process $\xi_{t}=\left(\ldots, \varepsilon_{t-1}, \varepsilon_{t}\right)$ consists of time series innovations $\varepsilon_{t} \sim$ iid $(0, \Sigma)$ as given Assumption LP above, and where $g(\cdot)$ is a measurable function such that $u_{t}$ is well-defined. Let $\left\{\varepsilon_{i}^{\prime}\right\}_{i \in \mathbb{Z}}$ be an iid copy of $\left\{\varepsilon_{i}\right\}_{i \in \mathbb{Z}}$. The following geometric moment contraction (GMC) condition holds, for all $t \in N$ with $M>0$ and $r \in(0,1)$,

$$
E\left[\left\|u_{t}-u_{t}^{*}\right\|^{q}\right] \leq M r^{t} \text {, with } q \geq 4
$$

where $u_{t}^{*}=g\left(\ldots, \varepsilon_{-1}^{\prime}, \varepsilon_{0}^{\prime}, \varepsilon_{1} \ldots, \varepsilon_{t-1}, \varepsilon_{t}\right)$.

Assumption LP, which follows MP, imposes conditional homoscedasticity, which may be restrictive in some financial applications. Assumption NLP is more general, includes assumption LP as a specific case, and allows various forms of stationary dependent processes such as GARCH/ARCH and threshold AR processes. As discussed below both LP and NLP conveniently allow for a Beveridge Nelson (BN; Phillips and Solo, 1972) decomposition of $u_{t}$. The general NLP case includes, in particular, GARCH processes but surprisingly does not incur any substantial cost in terms of the main derivations compared to proofs that rely directly on the LP conditions. The GMC condition imposed above is a sufficient condition enabling a systematic asymptotic theory for processes 
of the form (2.3) - see $\mathrm{Wu}$ (2011), where the asymptotics of many nonlinear time series under this condition are provided. The summability condition $\sum\left\|u_{t}-u_{t}^{*}\right\|<\infty$ is clearly met under the given GMC condition and this is analogous to the condition $\sum_{j=0}^{\infty} j\left\|F_{j}\right\|<\infty$ under LP. For the (I5) case, the LP assumption is needed for the proof of Lemma 2.2, where the same uniform approximation under NLP is also provided.

Under Assumption LP it is well known that there exists a valid BN decomposition, which leads to the following component-wise expressions (Phillips and Solo, 1992)

$$
\begin{aligned}
u_{t} & =F(1) \varepsilon_{t}-\triangle \tilde{\varepsilon}_{t}, \tilde{\varepsilon}_{t}=\sum_{j=0}^{\infty} \tilde{F}_{j} \varepsilon_{t-j}, \tilde{F}_{j}=\sum_{s=j+1}^{\infty} F_{s}, \\
u_{0 t} & =F_{0}(1) \varepsilon_{t}-\triangle \tilde{\varepsilon}_{0 t}, u_{x t}=F_{x}(1) \varepsilon_{t}-\triangle \tilde{\varepsilon}_{x t} .
\end{aligned}
$$

Under Assumption NLP, we have the related nonlinear BN decomposition (Lee, 2015)

$$
\begin{aligned}
& u_{t}=D_{t}-\triangle \tilde{D}_{t}=\left[\begin{array}{c}
D_{0 t} \\
D_{x t}
\end{array}\right]-\triangle\left[\begin{array}{c}
\tilde{D}_{0 t} \\
\tilde{D}_{x t}
\end{array}\right], \\
& D_{t}=\sum_{i=t}^{\infty} \mathcal{P}_{t} u_{i}, \tilde{D}_{t}=\sum_{k=1}^{\infty}\left(E\left[u_{t+k} \mid \mathcal{F}_{t}\right]\right)
\end{aligned}
$$

with $\mathcal{P}_{t} X:=E\left[X \mid \mathcal{F}_{t}\right]-E\left[X \mid \mathcal{F}_{t-1}\right]$, which follows the general martingale decomposition of a stationary time series (Hall and Heyde, 1980, chapter 5).

The long run covariance matrices associated with $u_{t}$ of LP are denoted as

$$
\begin{aligned}
& \Omega=\sum_{h=-\infty}^{\infty} E\left(u_{t} u_{t-h}^{\prime}\right)=F(1) \Sigma F(1)^{\prime}, \Delta=\sum_{h=0}^{\infty} E\left(u_{t} u_{t-h}^{\prime}\right), \\
& \Omega=\left[\begin{array}{ll}
\Omega_{00} & \Omega_{0 x} \\
\Omega_{x 0} & \Omega_{x x}
\end{array}\right], \Delta=\left[\begin{array}{ll}
\Delta_{00} & \Delta_{0 x} \\
\Delta_{x 0} & \Delta_{x x}
\end{array}\right] .
\end{aligned}
$$

Correspondingly, for NLP $u_{t}$ we define (c.f. Hall and Heyde, 1980, theorem 5.3)

$$
\Omega=\left\|\sum_{t=0}^{\infty} \mathcal{P}_{0} u_{t}\right\|_{2}^{2}=\left[\begin{array}{ll}
\Omega_{00} & \Omega_{0 x} \\
\Omega_{x 0} & \Omega_{x x}
\end{array}\right]
$$

Under (2.2) or (2.3) we have the functional law (Phillips and Solo, 1992):

$$
\frac{1}{\sqrt{n}} \sum_{j=1}^{\lfloor n s\rfloor} u_{j}:=\frac{1}{\sqrt{n}} \sum_{j=1}^{\lfloor n s\rfloor}\left[\begin{array}{l}
u_{0 t} \\
u_{x t}
\end{array}\right]=:\left[\begin{array}{c}
B_{0 n}(s) \\
B_{x n}(s)
\end{array}\right] \Longrightarrow\left[\begin{array}{c}
B_{0}(s) \\
B_{x}(s)
\end{array}\right]=B M\left[\begin{array}{ll}
\Omega_{00} & \Omega_{0 x} \\
\Omega_{x 0} & \Omega_{x x}
\end{array}\right],
$$

where $B=\left(B_{0}^{\prime}, B_{x}^{\prime}\right)^{\prime}$ is vector Brownian motion $(B M)$; and the following local to unity limit law 
for cases (I1), (I2) and (I4) also holds (Phillips, 1987):

$$
\frac{x_{\lfloor n r\rfloor}}{\sqrt{n}} \Longrightarrow J_{x}^{c}(r), \text { where } J_{x}^{c}(r)=\int_{0}^{r} e^{(r-s) C} d B_{x}(s),
$$

encompassing the (I1) case, where $J_{x}^{c}(r)=B_{x}(r)$ if $C=0$.

\section{$2.2 \quad$ IVX Construction}

Under rather general conditions, MP showed that the limit theory of the IVX estimator of $A$ is mixed normal and that suitably self-normalized test statistics have an asymptotic chi-square distribution. Since the limit theory is pivotal and therefore free of the nuisance parameter $C$, no information on the degree of regressor persistence is needed to execute tests as long as the regressors fall into the categories (I1), (I2) or (I3). The key step in IVX is the construction of an instrument using only endogenous information available in the regressors $\left\{x_{t}\right\}$ - hence the terminology IVX:

$$
\tilde{z}_{t}=\sum_{j=1}^{t} R_{n z}^{t-j} \triangle x_{j}, \quad \text { where } R_{n z}=I_{K}+\frac{C_{z}}{n^{\beta}}, \beta \in(0,1), C_{z}<0 .
$$

Since $\triangle x_{j}=\frac{C}{n^{\alpha}} x_{j-1}+u_{x j}$, we have the decomposition $\tilde{z}_{t}=\sum_{j=1}^{t} R_{n z}^{t-j} u_{x j}+\frac{C}{n^{\alpha}} \sum_{j=1}^{t} R_{n z}^{t-j} x_{j-1}$ denoted as

$$
\tilde{z}_{t}=z_{t}+\frac{C}{n^{\alpha}} \psi_{n t}
$$

The rate parameter $\beta$ and (diagonal) matrix $C_{z}$ in 2.9 are selected to ensure that $z_{t}$ is mildly integrated, which assures in turn that $z_{t}$ is less persistent than a unit root or local to unity regressor $x_{t}$. Using conventional observation matrix notation, the bias corrected IVX estimator of $A$ suggested by MP and its estimation error have the form

$$
\begin{aligned}
\tilde{A}_{n} & =\left(Y^{\prime} \tilde{Z}-n \widehat{\Delta}_{0 x}\right)\left(X^{\prime} \tilde{Z}\right)^{-1} \\
\tilde{A}_{n}-A & =\left(U_{0}^{\prime} \tilde{Z}-n \widehat{\Delta}_{0 x}\right)\left(X^{\prime} \tilde{Z}\right)^{-1}
\end{aligned}
$$

where $\widehat{\Delta}_{0 x}$ is some consistent estimate of the one sided long run covariance matrix $\Delta_{0 x}$. The estimator $\tilde{A}_{n}$ is a simple adjusted version of the conventional IV estimator $\hat{A}=\left(Y^{\prime} \tilde{Z}\right)\left(X^{\prime} \tilde{Z}\right)^{-1}$ using instruments $\tilde{z}_{t}$. In view of the decomposition in $2.10, z_{t}$ plays the role of a latent mildly integrated instrument and the remainder $\frac{C}{n^{\alpha}} \psi_{n t}$ is eliminated asymptotically due to its scaling coefficient $\frac{C}{n^{\alpha}}$. As a result we have nuisance parameter $(C)$ free inference using $\tilde{A}_{n}$. Martingale limit theory applies to the numerator matrix $U_{0}^{\prime} \tilde{Z}-n \widehat{\Delta}_{0 x}$ in 2.12, and this leads to a mixed normal limit theory that is well suited to inference.

\subsection{Limit Theory for Regressors with (Explosive) Local Unit Roots}

It turns out that IVX regression limit theory may be extended to explosive cases. The following result holds for the IVX estimator (2.11) with (I4) and local unit roots on the explosive side of 
unity. The limit theory under this (I4) case remains exactly the same as for the near integrated case (I2) or case N(ii) of MP.

Theorem 2.1 (Local Unit Roots on the Explosive Side) With $\beta \in(1 / 2,1)$,

$$
\operatorname{vec}\left[n^{\frac{1+\beta}{2}}\left(\tilde{A}_{n}-A\right)\right] \Longrightarrow M N\left(0,\left(\tilde{\Psi}_{x x}^{-1}\right)^{\prime} C_{z} V_{z z}^{x} C_{z} \tilde{\Psi}_{x x}^{-1} \otimes \Omega_{00}\right)
$$

where

$$
\tilde{\Psi}_{x x}=\Omega_{x x}+\int_{0}^{1} J_{x}^{c}(r) d J_{x}^{c}(r)^{\prime} \text { and } V_{z z}^{x}=\int_{0}^{\infty} e^{p C_{z}} \Omega_{x x} e^{p C_{z}} d p
$$

\subsection{Limit Theory for Regressors with Mildly Explosive Roots}

The asymptotics in the (I5) case turn out to be more complicated because the remainder term $\frac{C}{n^{\alpha}} \psi_{n t}$ now dominates the latent instrument $z_{t}$ in 2.10 and this has a substantial effect on the derivations, as discussed below. Moreover, it is not necessary to include the bias adjustment in this case and the limit theory for $\tilde{A}_{n}$ is identical to that of the unadjusted $\hat{A}=\left(Y^{\prime} \tilde{Z}\right)\left(X^{\prime} \tilde{Z}\right)^{-1}$. To present the limit theory concisely, we omit the bias correction term in this Section. In practice the bias corrected version (2.11) would be used since no precise knowledge about the various forms (I1)-(I5) cases is assumed. The limit theory remains unchanged as explained in Remark 2.5. Interestingly, the limit theory for $\hat{A}$ then coincides with that of the OLS estimator (see Theorem 4.1 in Magdalinos and Phillips (2009a)).

We consider case (I5) for mildly explosive regressors with common localizing exponent $\alpha \in(0,1)$ and localizing coefficient matrix $C>0$, combined with the moment condition $E\left\|\varepsilon_{1}\right\|^{q}<\infty, q \geq 4$. The following lemmas provide limit theory for standardized versions of the regressor $x_{t}$ that are needed for the asymptotic development. A rate condition on $\alpha\left(\alpha>\frac{2}{q}\right)$ is imposed in the second lemma to ensure a uniform strong approximation, ensuring that a wider range of $\alpha$ is admissible when higher moment conditions apply (see the discussion in the Proof of Lemma 2.2).

We start with the following lemma from Magdalinos and Phillips (2009a, lemma 4.1):

Lemma 2.1 Define $Y_{C n}:=\frac{1}{n^{\alpha / 2}} \sum_{j=1}^{k_{n}} R_{n}^{-j} F_{x}(1) \varepsilon_{j}$ for $k_{n} \rightarrow \infty$ such that $\left\|R_{n}\right\|^{-k_{n}} \rightarrow 0$. Then

$$
n^{-\alpha / 2} R_{n}^{-n} x_{n}=\frac{1}{n^{\alpha / 2}} \sum_{j=1}^{n} R_{n}^{-j} u_{x j}=\frac{1}{n^{\alpha / 2}} \sum_{j=1}^{k_{n}} R_{n}^{-j} u_{x j}+o_{p}(1)=: Y_{C n}+o_{p}(1)
$$

and

$$
Y_{C n} \Longrightarrow Y_{C} \equiv N\left(0, \int_{0}^{\infty} e^{-p C} \Omega_{x x} e^{-p C} d p\right)
$$

The requirement $\left\|R_{n}\right\|^{-k_{n}} \rightarrow 0$ in lemma 2.1 implies that $\frac{k_{n}}{n^{\alpha}} \rightarrow \infty$. As in MP (2009a), the quantities $Y_{C n}$ and $Y_{C}$ play an important role in the asymptotic theory. In addition, the following uniform approximation holds, which helps to simplify proofs. A similar approximation was shown for the scalar case in Phillips and Magdalinos (2007b). 
Lemma 2.2 (Uniform Approximation) With the same $k_{n}$ defined in lemma 2.1 and $j_{n}=j-1$, there exist random variables $\tilde{Y}_{C n, j_{n}}$ and $\tilde{Y}_{C}$ in a suitably expanded common probability space such that

$$
\sup _{j_{n} \text { such that } k_{n} \leq j_{n} \leq n}\left\|\tilde{Y}_{C n, j_{n}}-\tilde{Y}_{C}\right\|=o_{p}(1)
$$

where $\tilde{Y}_{C n, j_{n}}$ and $\tilde{Y}_{C}$ are distributionally equivalent versions of $Y_{C n, j_{n}}=\frac{1}{n^{\alpha / 2}} R_{n}^{-j_{n}} x_{j_{n}}$ and $Y_{C}$, so that $\tilde{Y}_{C n, j_{n}}={ }_{d} Y_{C n, j_{n}}$ and $\tilde{Y}_{C}={ }^{d} Y_{C}$.

Remark 2.1 The above uniformly strong approximation of the normalized process of a vector of mildly explosive regressors is particularly useful in developing limit theory in mildly explosive regression. But for the purpose of our proof here convergence in probability is enough and for that result somewhat weaker moment conditions may be used (see lemma 3.1 of Phillips, 2007).

For the sequence $k_{n}$ used in lemmas 2.1 and 2.2, we have $\frac{k_{n}}{n^{\alpha}} \rightarrow \infty$ and it follows that when $t<$ $k_{n},\left\|n^{-\alpha / 2} R_{n}^{-t} x_{t}\right\|_{L_{2}}$ either degenerates to zero (when $t=o\left(n^{\alpha}\right)$ ) or is bounded (when $t=O\left(k_{n}\right)$ ). Combined with lemma 2.2, we have

$$
\sup _{1 \leq t \leq n}\left\|n^{-\alpha / 2} R_{n}^{-t} x_{t}\right\|_{L_{2}}<\infty
$$

A similar uniform approximation to that of lemma 2.2 holds for $\psi_{n t}$ in 2.10, with a slight modification to the index range of $t$ as shown in the following lemma.

Lemma 2.3 For $k_{n}$ and $k_{n}^{\prime}$ satisfying the rate condition $\frac{n^{\alpha \vee \beta}}{k_{n}}+\frac{n^{\alpha \wedge \beta}}{k_{n}^{\prime}} \rightarrow 0$ where $\alpha \vee \beta=\max (\alpha, \beta)$ and $\alpha \wedge \beta=\min (\alpha, \beta)$, we have

$$
\frac{1}{n^{\frac{\alpha}{2}+\alpha \wedge \beta}} R_{n}^{-t} \psi_{n t}=C_{z \alpha \beta} \tilde{Y}_{C}+o_{p}(1)
$$

for all $t \in\left[k_{n}+k_{n}^{\prime}, n\right]$ where

$$
C_{z \alpha \beta}:=\left\{\begin{array}{cc}
-C_{z}^{-1}, & \text { if } \beta<\alpha \\
C^{-1}, & \text { if } \alpha<\beta \\
\left(C-C_{z}\right)^{-1}, & \text { if } \alpha=\beta
\end{array} .\right.
$$

Remark 2.2 As the proof of the lemma in the Appendix reveals, the index set $\left[k_{n}+k_{n}^{\prime}, n\right]$ for $t$ ensures the negligibility of certain frontal sums involving the standardized components of $\psi_{n t}$. As will become clear, the condition is used only in the proofs of the intermediate lemmas and does not appear in the main results because the full sums are dominated by the tail summation. Note that even for moderate sample sizes $n$, the set is well defined. For example, when $n=30, \alpha \vee \beta=0.8$ and $\alpha \wedge \beta=0.6$, we have $n-n^{0.85}-n^{0.65}>0$ so the index set is not empty and evidently has $O(n)$ observations as $n \rightarrow \infty$.

Remark 2.3 In 2.14) the standardization by $n^{\frac{\alpha}{2}+\alpha \wedge \beta}$ involves (potentially) both localizing coefficients $\alpha$ and $\beta$ (depending on their respective magnitudes). The intuition for this standardization 
is that the quantity $\psi_{n t}=\sum_{j=1}^{t} R_{n z}^{t-j} x_{j-1}=\sum_{j=1}^{t}\left\{R_{n z}^{t-j} R_{n}^{(j-1)}\right\} R_{n}^{-(j-1)} x_{j-1}$ involves the weighting matrices $R_{n z}^{t-j}$ which downweight the components $x_{j-1}$ in the sum, whereas these components themselves, being mildly explosive, are weighted by $R_{n}^{-(j-1)}$, which leads to an interacting weighting system involving the matrices $R_{n z}^{t-j} R_{n}^{(j-1)}$. Upon summation these weights may be dominated by the near stationary components that involve $R_{n z}($ and $\beta$ ) or the mildly explosive components that involve $R_{n}$ (and $\alpha$ ).

Remark 2.4 In consequence, both localizing coefficients $\alpha$ and $\beta$ appear in the component limit theory for the numerator and denominator matrices of the IVX estimator (see lemma 2.4 below). Intuitively, if $\beta$ is smaller than $\alpha$, then the instruments $z_{t}$ are near stationary and these nearstationary instruments tend to attenuate the mildly explosive behavior of $x_{t}$ in the IV regression, thereby leading to the factor $n^{\alpha \wedge \beta}$ in the normalization and slowing down the rate of convergences in the components.

As in 2.13, when $t<k_{n}+k_{n}^{\prime}$, it can be shown that $\left\|n^{-(\alpha / 2+(\alpha \wedge \beta))} R_{n}^{-t} \psi_{n t}\right\|_{L_{2}}$ is either degenerate or bounded. So we have

$$
\sup _{1 \leq t \leq n}\left\|n^{-(\alpha / 2+(\alpha \wedge \beta))} R_{n}^{-t} \psi_{n t}\right\|_{L_{2}}<\infty
$$

With these results in hand, the limit theory follows for the numerator and denominator of the IVX estimator under (I5) mildly explosive regressors. The stronger signal in the remainder terms results in a new limit theory that involves the nuisance parameters $C$ and $C_{z \alpha \beta}$.

Lemma 2.4 (IVX Numerator and Denominator) Define $\tilde{\Psi}_{y y}:=\int_{0}^{\infty} e^{-p C} Y_{C} Y_{C}^{\prime} e^{-p C} d p$.

(1) $\operatorname{vec}\left(\frac{1}{n^{(\alpha \wedge \beta)}} \sum_{t=1}^{n} u_{0 t} \tilde{z}_{t}^{\prime} R_{n}^{-n}\right) \Longrightarrow\left(C C_{z \alpha \beta} \otimes I_{m}\right) \times M N\left(0, \tilde{\Psi}_{y y} \otimes \Omega_{00}\right)$.

(2) $\frac{1}{n^{\alpha+(\alpha \wedge \beta)}} \sum R_{n}^{-n} x_{t} \tilde{z}_{t}^{\prime} R_{n}^{-n} \Longrightarrow \tilde{\Psi}_{y y} \times\left(C C_{z \alpha \beta}\right)$.

Remark 2.5 The limit theory of the numerator given in lemma 2.4-(1) shows that $\sum_{t=1}^{n} u_{0 t} z_{t}^{\prime}$ becomes asymptotically negligible in relation to the term $\sum_{t=1}^{n} u_{0 t} \psi_{n t}^{\prime}$, which is usually a (negligible) remainder, and it is this term that dominates the asymptotics. Moreover, unlike the other cases (I1)-(I4), we no longer need the built-in serial correlation bias correction associated with the $O_{p}(n)$ sample covariance term $\sum_{t=1}^{n} u_{0 t} z_{t}^{\prime}$ and an estimate of the corresponding one sided long run covariance matrix. A similar property arises in the case of OLS estimation under mildly explosive regressors (Magdalinos and Phillips, 2009a).

The limit theory of the IVX estimator 2.11) under (I5) is therefore equivalent to that of the OLS estimator in Magdalinos and Phillips (2009a, theorem 4.1). 
Theorem 2.2 (Mildly Explosive Regressor) With $\alpha \in(2 / q, 1)$ and $\beta \in(1 / 2,1)$,

$$
\operatorname{vec}\left[n^{\alpha}\left(\tilde{A}_{n}-A\right) R_{n}^{n}\right] \Longrightarrow M N\left(0, \tilde{\Psi}_{y y}^{-1} \otimes \Omega_{00}\right)
$$

where

$$
\tilde{\Psi}_{y y}=\int_{0}^{\infty} e^{-p C} Y_{C} Y_{C}^{\prime} e^{-p C} d p, \text { and } Y_{C} \equiv N\left(0, \int_{0}^{\infty} e^{-p C} \Omega_{x x} e^{-p C} d p\right)
$$

The results of the limit theory given in theorem 2.1 and theorem 2.2 for the LUR explosive and mildly explosive cases depend on the unknown localizing coefficient matrix $C$ and the unknown rate parameter $\alpha$. So these results are not feasible for practical work as they stand. However,self normalized versions of the statistics have a chi-square limit theory in both the (I4) and (I5) cases, providing a convenient basis for inference.

To illustrate, we follow the usual (linear) hypothesis testing framework in which $H$ is a known $r \times m K$ matrix of rank $r$ and $h$ is a known vector. Obvious extensions hold for general analytic restrictions.

Theorem 2.3 (LUR Explosive or Mildly Explosive Regressor) Under $H_{0}:$ Hvec $(A)=h$ with $\alpha \in(2 / q, 1)$ and $\beta \in(1 / 2,1)$,

$$
\left(H \operatorname{vec}\left(\tilde{A}_{n}\right)-h\right)^{\prime}\left[H\left\{\left(X^{\prime} P_{\tilde{Z}} X\right)^{-1} \otimes \hat{\Omega}_{00}\right\} H^{\prime}\right]^{-1}\left(H v e c\left(\tilde{A}_{n}\right)-h\right) \Longrightarrow \chi^{2}(r),
$$

where

$$
\left(X^{\prime} P_{\widetilde{Z}} X\right)^{-1}=\left\{\left(\sum_{t=1}^{n} x_{t} \tilde{z}_{t}^{\prime}\right)\left(\sum_{t=1}^{n} \tilde{z}_{t} \tilde{z}_{t}^{\prime}\right)^{-1}\left(\sum_{t=1}^{n} x_{t} \tilde{z}_{t}^{\prime}\right)^{\prime}\right\}^{-1} .
$$

Although the limit theory of the IVX estimator under (I5) mildly explosive regressors differs from the other (I1)-(I4) cases, the usual chi-square limit theory for the self-normalized test statistic still holds. Theorem 2.3, taken together with theorem 3.8 of MP, therefore shows that IVX regression leads to a single inference procedure in all cases (I1)-(I5). The broad range of this limit theory is helpful in empirical work where there is inevitable uncertainty about the degree of persistence in the regressors. Importantly, however, the limit theory in Theorem 2.3 requires $x_{t}$ to share the same localizing exponent $\alpha \in(0,1)$. Some relaxation of this condition is explored in Section 3 where we allow for both mildly explosive and mildly integrated regressors.

\subsection{Regressions with Intercept}

Practical implementations of regressions such as 2.1) commonly involved deterministic components such as intercepts or trends. In predictive regression versions of 2.1], it is especially common to include an intercept in empirical applications. In their recent study of such regressions, Kostakis et al. (2014) allow for the presence of an intercept and show that the first order IVX limit theory 
remains the same as MP for cases (I1)-(I3). The (I4) case is essentially the same as the (I2) case considered in Kostakis et al (2014) upon working with a demeaned OU process. This leaves case (I5) which we now consider.

We follow the same procedure as in Kostakis et al (2014) of including an intercept in (2.1) and using the undemeaned IVX instruments, so that (2.1) is replaced by the system

$$
\begin{aligned}
& y_{t}=\mu+A x_{t}+u_{0 t}, \\
& x_{t}=R_{n} x_{t-1}+u_{x t},
\end{aligned}
$$

Defining $x_{t}^{\mu}=x_{t}-n^{-1} \sum x_{t}, y_{t}^{\mu}=y_{t}-n^{-1} \sum y_{t}$ and $u_{0 t}^{\mu}=u_{0 t}-n^{-1} \sum u_{0 t}$, demeaning (2.16) leads to $y_{t}^{\mu}=A x_{t}^{\mu}+u_{0 t}^{\mu}$. Denote the corresponding observation matrices as $Y^{\mu}, X^{\mu}$ and $U_{0}^{\mu}$, respectively. Then IVX estimation in the (I5) case, where bias correction does not play any role and is therefore omitted for ease of exposition, leads to the IVX estimator

$$
\tilde{A}_{n}^{\mu}=\left(Y^{\mu^{\prime}} \tilde{Z}\right)\left(X^{\mu^{\prime}} \tilde{Z}\right)^{-1}=A+\left(U_{0}^{\mu \prime} \tilde{Z}\right)\left(X^{\mu \prime} \tilde{Z}\right)^{-1}
$$

The effect of demeaning, or estimating an intercept in the regression, becomes relevant only in the limit theory of the sample covariance component $X^{\mu^{\prime}} \tilde{Z}=\sum x_{t} \tilde{z}_{t}^{\prime}$ (Lemma 2.4-(2)). Upon standardization, this component behaves to the first order just as it does in the case where an intercept is not fitted, as shown in the following proposition.

Proposition 2.4 For the (I5) case,

$$
\frac{1}{n^{\alpha+(\alpha \wedge \beta)}} \sum R_{n}^{-n} x_{t}^{\mu} \tilde{z}_{t}^{\prime} R_{n}^{-n}=\frac{1}{n^{\alpha+(\alpha \wedge \beta)}} \sum R_{n}^{-n} x_{t} \tilde{z}_{t}^{\prime} R_{n}^{-n}+o_{p}(1) .
$$

In the same way, the first order limit theory of this sample covariance remains unchanged in the (I4) case. Hence, the effects of including an intercept in the system regression is innocuous, at least in first order asymptotics. Simulations conducted by Kostakis et al (2014) show that the inclusion of an intercept can produce finite sample size distortion in Wald statistic tests in some cases. They also show that a simple correction to the $\tilde{Z}^{\prime} \tilde{Z}$ signal matrix that appears in the Wald statistic estimated variance matrix of the IVX estimator is successful in reducing this distortion. Readers are referred to their paper for details and further discussion.

\section{IVX Regression with Mixed Roots}

The results above combined with those of MP show that the IVX approach is applicable for regressors with common roots in a wide vicinity of unity including all (I1) - (I5) cases. We might also expect the same method to be valid when there are mixed degrees of persistence in the regressors, which is likely in some empirical work (e.g. Campbell and Yogo, 2006). This section confirms that conjecture, showing the same limit theory given in theorem 2.2 holds when there are multiple regressors with mixed roots in the vicinity of unity. 
For exposition, it is convenient to consider the prototypical model with $m=1$ and $K=2$ in (2.1) so that $y_{t}$ is scalar and $x_{t}$ is a bivariate $\mathrm{AR}(1)$ process with mixed roots. The remaining restrictive element implied by this bivariate regressor system for the general case where there may be many regressors is commonality of the mildly explosive and mildly integrated roots. The simplified system has the form

$$
\begin{aligned}
& y_{t}=a^{\prime} x_{t}+u_{0 t}, a^{\prime}=\left[\begin{array}{ll}
a_{1} & a_{2}
\end{array}\right], x_{t}=\left[\begin{array}{l}
x_{1 t} \\
x_{2 t}
\end{array}\right], \\
& x_{t}=R_{n} x_{t-1}+u_{x t}, R_{n}=\left[\begin{array}{cc}
\rho_{n} & 0 \\
0 & \theta_{n}
\end{array}\right], u_{x t}=\left[\begin{array}{l}
u_{x 1 t} \\
u_{x 2 t}
\end{array}\right] .
\end{aligned}
$$

We impose different degrees of persistence in the two regressors with the following specification:

$$
\begin{aligned}
& \rho_{n}=1+\frac{c_{1}}{n^{\alpha_{1}}}, \text { where } c_{1} \in\left\{\begin{array}{cc}
(-\infty, 0), & \text { if } \alpha_{1} \in(0,1) \\
(-\infty, \infty), & \text { if } \alpha_{1}=1
\end{array},\right. \\
& \theta_{n}=1+\frac{c_{2}}{n^{\alpha_{2}}}, \text { where } c_{2} \in(0, \infty) \text { and } \alpha_{2} \in(0,1) .
\end{aligned}
$$

Accordingly, $x_{1 t}$ falls under one of the specifications (I1)-(I4), while $x_{2 t}$ is a mildly explosive regressor corresponding to $(\mathrm{I} 5)^{3}$. Here we demonstrate the robustness of IVX estimation for the system parameter $a$ in (3.1) in a mixed root regressor environment.

From the decomposition $(2.4)$ we may express $u_{t}$ in component form as

$$
u_{t}=\left[\begin{array}{c}
u_{0 t} \\
u_{x 1 t} \\
u_{x 2 t}
\end{array}\right]=\left[\begin{array}{c}
F_{0}(1)_{1 \times 3} \\
F_{x 1}(1)_{1 \times 3} \\
F_{x 2}(1)_{1 \times 3}
\end{array}\right]\left[\begin{array}{c}
\varepsilon_{0 t} \\
\varepsilon_{x 1 t} \\
\varepsilon_{x 2 t}
\end{array}\right]-\triangle\left[\begin{array}{c}
\tilde{\varepsilon}_{0 t} \\
\tilde{\varepsilon}_{x 1 t} \\
\tilde{\varepsilon}_{x 2 t}
\end{array}\right],
$$

under the same assumptions as $(2.2)$. The long run variance matrices and the limit theory are the same as in (2.6), 2.7) and (2.8) except that the subscripts 0,1 and 2 now signify $u_{0}, u_{x 1}$ and $u_{x 2}$, respectively. The IVX instrument is constructed in the same way as 2.9 with $C_{z}=\operatorname{diag}\left(c_{z 1}, c_{z 2}\right)<$ 0 and $\beta \in(1 / 2,1)$.

The bias corrected IVX estimator of $a$ has estimation error

$$
\begin{aligned}
\tilde{a}-a & =\left[\begin{array}{c}
\tilde{a}_{1}-a_{1} \\
\tilde{a}_{2}-a_{2}
\end{array}\right]=\left(\tilde{Z}^{\prime} X\right)^{-1}\left(\tilde{Z}^{\prime} U_{0}-n \hat{\Delta}_{x 0}\right) \\
& =\left[\begin{array}{cc}
\sum_{t=1}^{n} \tilde{z}_{1 t} x_{1 t} & \sum_{t=1}^{n} \tilde{z}_{1 t} x_{2 t} \\
\sum_{t=1}^{n} \tilde{z}_{2 t} x_{1 t} & \sum_{t=1}^{n} \tilde{z}_{2 t} x_{2 t}
\end{array}\right]^{-1}\left[\begin{array}{c}
\sum_{t=1}^{n} \tilde{z}_{1 t} u_{0 t}-n \hat{\Delta}_{01} \\
\sum_{t=1}^{n} \tilde{z}_{2 t} u_{0 t}-n \hat{\Delta}_{02}
\end{array}\right] .
\end{aligned}
$$

\footnotetext{
${ }^{3}$ Dual manifestations of nonstationarity with different roots of this type were first considered in a 2010 Yale Take Home Examination by the first author. The idea developed later into Phillips and Lee (2015b) who considered inference about the roots in a vector autoregression of the type 3.2 .
} 
We employ the following normalizing matrices to accommodate the different orders of magnitude

$$
D_{n}=\left[\begin{array}{cc}
n^{\frac{1+\left(\alpha_{1} \wedge \beta\right)}{2}} & 0 \\
0 & \theta_{n}^{n} n^{\alpha_{2} \wedge \beta}
\end{array}\right], C_{n}=\left[\begin{array}{cc}
n^{\frac{1+\left(\alpha_{1} \wedge \beta\right)}{2}} & 0 \\
0 & \theta_{n}^{n} n^{\alpha_{2}}
\end{array}\right]
$$

Then

$$
C_{n}(\tilde{a}-a)=\left(D_{n}^{-1} \tilde{Z}^{\prime} X C_{n}^{-1}\right)^{-1} D_{n}^{-1}\left(\tilde{Z}^{\prime} U_{0}-n \hat{\Delta}_{x 0}\right)
$$

To show the joint convergence of $n^{\frac{1+\left(\alpha_{1} \wedge \beta\right)}{2}}\left(\tilde{a}_{1}-a_{1}\right)$ and $\theta_{n}^{n} n^{\alpha_{2}}\left(\tilde{a}_{2}-a_{2}\right)$, we first show the asymptotic independence of these two components. As in Magdalinos and Phillips (2009a), the asymptotic behavior of $\theta_{n}^{n} n^{\alpha_{2}}\left(\tilde{a}_{2}-a_{2}\right)$ is determined by two independent asymptotic Gaussian processes

$$
Y_{n}=\left[\begin{array}{c}
Y_{c_{0} n} \\
Y_{c_{2} n}
\end{array}\right]:=\left[\begin{array}{c}
\frac{1}{n_{2}} \sum_{t=k_{n}+1}^{n} \theta_{n}^{-(n-t)} F_{0}(1) \varepsilon_{t} \\
\frac{1}{n^{\frac{\alpha}{2}}} \sum_{j=1}^{k_{n}} \theta_{n}^{-j} F_{x 2}(1) \varepsilon_{j}
\end{array}\right] \Longrightarrow\left[\begin{array}{c}
Y_{c_{0}} \\
Y_{c_{2}}
\end{array}\right] \equiv N\left(0_{2 \times 1},\left[\begin{array}{cc}
\frac{\Omega_{00}}{2 c_{2}} & 0 \\
0 & \frac{\Omega_{22}}{2 c_{2}}
\end{array}\right]\right)
$$

From MP, the vector martingale $\sum_{t=1}^{n} \xi_{n t}$ with

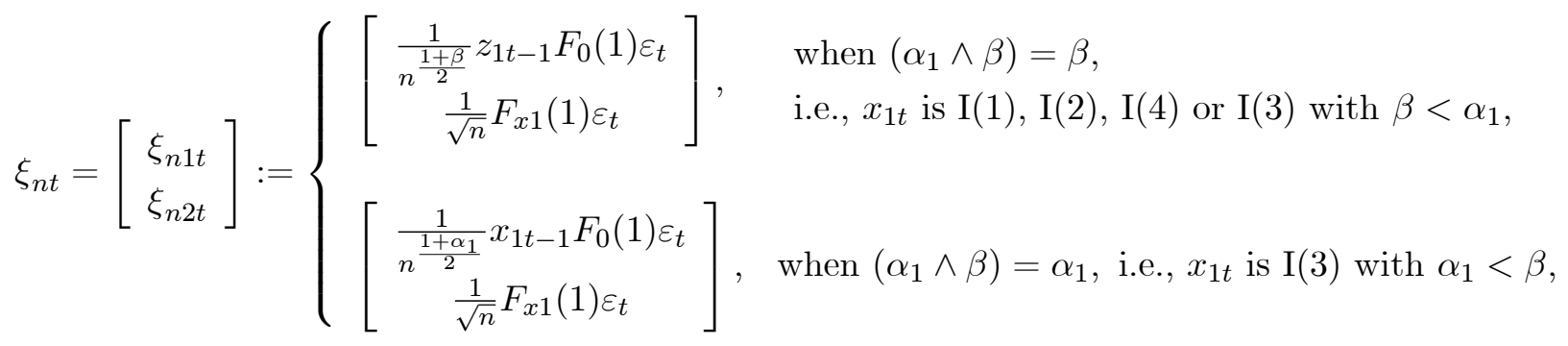

determines the Gaussian limit theory of $n^{\frac{1+\left(\alpha_{1} \wedge \beta\right)}{2}}\left(\tilde{a}_{1}-a_{1}\right)$. When $\left(\alpha_{1} \wedge \beta\right)=\beta$, the asymptotic independence between $\sum_{t=1}^{n} \xi_{n 1 t}$ and $\sum_{t=1}^{n} \xi_{n 2 t}$ is shown in proposition A1 in MP. The same proof also holds for the $\left(\alpha_{1} \wedge \beta\right)=\alpha_{1}$ case. The joint convergence of $n^{\frac{1+\left(\alpha_{1} \wedge \beta\right)}{2}}\left(\tilde{a}_{1}-a_{1}\right)$ and $\theta_{n}^{n} n^{\alpha_{2}}\left(\tilde{a}_{2}-a_{2}\right)$ is therefore achieved by showing the asymptotic independence between $Y_{n}$ and $\sum_{t=1}^{n} \xi_{n t}$, which is done in the following lemma.

Lemma 3.1 $Y_{n}$ is asymptotically independent of the vector martingale $\sum_{t=1}^{n} \xi_{n t}$.

The normalized denominator matrix is asymptotically diagonal under some mild rate conditions on $\beta$ and $\alpha_{i}(i=1,2)$, which enhances the development of the joint limit theory.

Lemma 3.2 Under the rate condition $\alpha_{1}+\alpha_{2}<1+\beta$, we have

$$
D_{n}^{-1} \tilde{Z}^{\prime} X C_{n}^{-1} \Longrightarrow\left[\begin{array}{cc}
\tilde{\Psi}_{11} & 0 \\
0 & c_{x z 2}(\alpha, \beta) c_{2} \tilde{\Psi}_{22}
\end{array}\right]
$$


where $\tilde{\Psi}_{22}=\frac{Y_{c_{2}}^{2}}{2 c_{2}}$, and

$$
\tilde{\Psi}_{11}=\left\{\begin{array}{cr}
\frac{1}{-c_{z 1}}\left\{\Omega_{11}+\int_{0}^{1} B_{1} d B_{1}\right\} & \text { if } x_{1 t} \text { is unit root : I(1), } \\
\frac{1}{-c_{z 1}}\left\{\Omega_{11}+\int_{0}^{1} J_{x}^{c 1} d J_{x}^{c 1}\right\} & \text { if } x_{1 t} \text { is local to unity : (I2) or (I4), } \\
\frac{1}{-2 c_{z 1}} \Omega_{11} & \text { if } x_{1 t} \text { is mildly integrated - (I3) with } \beta<\alpha_{1} \\
\frac{1}{-2\left(c_{1}+c_{z 1}\right)} \Omega_{11} & \text { if } x_{1 t} \text { is mildly integrated - (I3) with } \beta=\alpha_{1} \\
\frac{1}{-2 c_{1}} \Omega_{11} & \text { if } x_{1 t} \text { is mildly integrated - (I3) with } \alpha_{1}<\beta
\end{array} .\right.
$$

Remark 3.1 Note that $\alpha_{1}+\alpha_{2}<1+\beta$ may not hold in some cases. In particular, if we have a local-to-unity regressor $x_{1 t}\left(\alpha_{1}=1\right)$ and a mildly explosive regressor $x_{2 t}$ whose root is very close to being local to unity (i.e., $\alpha_{2}$ is close to unity), then $\alpha_{2}<\beta$ may not hold and we may not have a diagonal limit for the moment matrix $\tilde{Z}^{\prime} X$ upon standardization. For example, if $\alpha_{1}=1$ and $\beta<\alpha_{2}$, then $\alpha_{1}+\alpha_{2}<1+\beta$ fails. Intuitively, even though $x_{1 t}$ and $x_{2 t}$ have different orders of magnitude in this case, their asymptotic behavior is not distinct enough to ensure negligibility of the off diagonal elements when IVX leads to an instrument $\tilde{z}_{2 t}$ that is close to stationarity ( $\beta$ close to 0.5$)$. In such cases, the range of $\beta$ for which $\tilde{Z}^{\prime} X$ is diagonal asymptotically is restricted to the smaller region $\beta \in\left(\alpha_{2}, 1\right)$.

The limit theory of $C_{n}(\tilde{a}-a)$ is therefore obtained from the independent marginal convergence of the two components and the mixed roots affect each of these components separately in the limit.

Theorem 3.1 Under the rate conditions $\alpha_{1} \in(1 / 3,1)$ and $\beta \in\left(\left(\alpha_{2} \vee 2 / 3\right), 1\right)$,

$$
C_{n}(\tilde{a}-a) \Longrightarrow M N\left(0_{2 \times 1},\left[\begin{array}{cc}
\tilde{\Phi}_{11}^{-1} \Omega_{00} & 0 \\
0 & \tilde{\Psi}_{22}^{-1} \Omega_{00}
\end{array}\right]\right)
$$

where $\tilde{\Psi}_{22}=\frac{Y_{c_{2}}^{2}}{2 c_{2}}, Y_{c 2} \equiv N\left(0, \frac{\Omega_{22}}{2 c_{2}}\right)$ as in theorem 2.2, and $\tilde{\Psi}_{11}$ is given as

$$
\tilde{\Phi}_{11}^{-1}=\left\{\begin{array}{cc}
\left\{\frac{1}{-c_{z 1}}\left(\Omega_{11}+\int_{0}^{1} B_{1} d B_{1}\right)\right\}^{-2}\left(\frac{1}{-2 c_{z 1}} \Omega_{11}\right) & \text { if } x_{1 t} \text { is unit root - I(1) } \\
\left\{\frac{1}{-c_{z 1}}\left\{\Omega_{11}+\int_{0}^{1} J_{x}^{c 1} d J_{x}^{c 1}\right\}\right\}^{-2}\left(\frac{1}{-2 c_{z 1}} \Omega_{11}\right) & \text { if } x_{1 t} \text { is local to unity - (I2) or (I4) } \\
\left(\frac{1}{-2 c_{z 1}} \Omega_{11}\right)^{-1} & \text { if } x_{1 t} \text { is mildly integrated - (I3) with } \beta<\alpha_{1} \\
\frac{\left(c_{1}+c_{z 1}\right)^{2}}{c_{1}^{2}}\left(\frac{1}{-2 c_{1}} \Omega_{11}\right)^{-1} & \text { if } x_{1 t} \text { is mildly integrated - (I3) with } \beta=\alpha_{1} \\
\left(\frac{1}{-2 c_{1}} \Omega_{11}\right)^{-1} & \text { if } x_{1 t} \text { is mildly integrated - (I3) with } \alpha_{1}<\beta
\end{array}\right.
$$

Remark 3.2 The rate condition $\alpha_{1} \in(1 / 3,1)$ ensures that the numerator of IVX estimator of the first component $\sum_{t=1}^{n} \tilde{z}_{1 t} u_{0 t}-n \hat{\Delta}_{01}$ has an asymptotic normal distribution. If we replace the weakly dependent structure of $u_{0 t}$ with an iid or mds structure for $u_{0 t}$, as is common in predictive regressions, a wider region of $\alpha_{1}$ is possible (see Kostakis et al., 2014). The condition that $\beta \in$ 
$\left(\left(\alpha_{2} \vee 2 / 3\right), 1\right)$ is explained as follows. First, the requirement that the choice parameter $\beta$ exceeds $2 / 3$ accommodates consistent estimation of the long run covariance using $\hat{\Delta}_{x 0}$ (see Lemma AO in $M P)$. The condition that $\beta$ exceeds $\alpha_{2}$ ensures asymptotic diagonality of the denominator matrix of IVX (the rate condition in Lemma 3.2). Section 4 shows that reliable choices of $\beta$ are contained in this region.

Remark 3.3 Since $\tilde{\Psi}_{22}=\frac{Y_{c_{2}}^{2}}{2 c_{2}}$ and $Y_{c_{0}} \equiv N\left(0, \frac{\Omega_{00}}{2 c_{2}}\right)$ is independent of $Y_{c_{2}}$, we have

$$
\theta_{n}^{n} n^{\alpha_{2}}\left(\tilde{a}_{2}-a_{2}\right) \Longrightarrow 2 c_{2} \frac{Y_{c_{0}}}{Y_{c_{2}}} \equiv 2 c_{2}\left(\frac{\Omega_{00}}{\Omega_{22}}\right)^{1 / 2} \mathcal{C}
$$

where $\mathcal{C}$ is a standard Cauchy variate, giving the same result as that of Magdalinos and Phillips (2009a, remark 4.1a). This result again confirms the asymptotic equivalence of IVX and OLS estimation with mildly explosive regressors.

As anticipated, the same self-normalized test statistic continues to deliver an asymptotic chisquare test free of any nuisance parameters, at least for full vector tests.

Theorem 3.2 Under $H_{0}: a=a_{0}$ and the rate condition in Theorem 3.2,

$$
\left(\tilde{a}-a_{0}\right)^{\prime}\left[\left(X^{\prime} P_{\widetilde{Z}} X\right)^{-1} \hat{\Omega}_{00}\right]^{-1}\left(\tilde{a}-a_{0}\right) \Longrightarrow \chi^{2}(2),
$$

where $\left(X^{\prime} P_{\widetilde{Z}} X\right)^{-1}$ is defined in Theorem 2.3.

This result demonstrates considerable robustness of the IVX approach to mixed degrees of persistence in the regressors, thereby widening the ambit of empirical research covered by this procedure and providing a step towards a single valid procedure for inference that allows for a large class of persistent, but differently behaved regressors. More general cases that allow for multivariate regressors with multiple mixed roots are treated in the same way. For example, the process $x_{t}=R_{n} x_{t-1}+u_{x t}$ may have a coefficient matrix of the form

$$
R_{n}=\left[\begin{array}{cc}
\left(R_{1 n}\right)_{K_{1} \times 1} & 0 \\
0 & \left(R_{2 n}\right)_{K_{2} \times 1}
\end{array}\right],
$$

where $R_{1 n}$ has roots in the (I1)-(I4) class with common localizing exponent and $R_{2 n}$ involves mildly explosive coefficients of the form (I5) again with common localizing exponent. Analogous arguments to those in this section lead to asymptotic independence between suitably standardized versions of the processes for each group of regressors and the sample moment matrices $X^{\prime} \tilde{Z}$ and $\tilde{Z}^{\prime} \tilde{Z}$ will be asymptotically block diagonal after similar normalizations. Hence, in the same way as Theorem 3.2 we end up with a chi-square test that applies for a general class of mixed regressors.

While the theory presented here includes mixed regressors, it does not provide a fully general treatment that admits regressors with variable localizing exponents, either for the mildly integrated 
or the mildly explosive regressors. Similarly it does not provide a general limit theory of Wald testing with an arbitrary restriction matrix $H$ when there are mixed regressors and variable localizing exponents. Limit theory under completely agnostic conditions where there are multiple localizing exponents $\left\{\alpha_{k}: k=1, \ldots, K\right\}$ in $R_{n}$ and general restrictions to be tested is a much more challenging task and is the subject of ongoing research by Magdalinos and Phillips. The partial results provided by Theorem 3.2 are nonetheless useful in bivariate regression cases with possibly mixed degrees of persistence and show clearly the potential of the IVX methodology to encompass a wide range of persistence in the regressors.

\section{On the Choice of IVX Tuning Parameter}

Implementation of IVX estimation requires choice of the tuning parameter $\beta$ that is involved in the generation of the IVX instruments via (2.9). Evidently, larger values of $\beta$ generally produce higher rates of convergence (c.f. theorem 2.1) and more efficient test procedures may therefore be expected. On the other hand, the central idea of IVX instrumentation - filtering a persistent regressor to generate an instrument of less persistence, thereby ensuring validity of chi-squared test limit theory - suggests that we need to impose an upper bound for $\beta$ that is less than unity.

To fix ideas and keep the following discussion simple, we use the predictive regression setting that has been widely adopted in empirical finance, whereby the one period ahead dependent variable $y_{t+1}$ is used instead of $y_{t}$ in (2.1) and the regression error $u_{0 t+1}$ is assumed to be a martingale difference. These modifications do not change the limit theory presented earlier and correspond to the standard environment of existing studies, such as Campbell and Yogo (2006) and Jansson and Moreira (2006). The martingale difference structure on $u_{0 t+1}$ implies that there is no predictability of $y_{t+1}$ under the null hypothesis $H_{0}: a=0$. With this structure, the following DGP is imposed

$$
\begin{aligned}
y_{t+1} & =\mu+a^{\prime} x_{t}+u_{0 t+1}, u_{0 t+1} \sim \operatorname{mds}\left(0, \Sigma_{00}\right), \\
x_{t+1} & =\left(I_{K}+\frac{C}{n}\right) x_{t}+u_{x t+1},
\end{aligned}
$$

where $u_{x t+1}$ is a linear process generated as in (2.2). The AR dynamics of the predictive variables is formulated as in 2.1) with (I1)-(I6) specifications, describing the various degrees of persistence 4

\subsection{Failure of Standard MSE Choice Criteria}

Since larger values of $\beta$ improve convergence rates whereas bias effects enter and the IVX mixed normal limit theory fails when $\beta=1$, conventional asymptotic mean squared error (MSE) criteria (and associated cross validation approaches) might be expected to lead to suitable empirical choice criteria for $\beta$. As we now show, the asymptotic MSE criterion monotonically decreases as $\beta$ increases,

\footnotetext{
${ }^{4}$ Standard present value models in financial theory do not usually admit explosive process solutions, since these are typically ruled out by transversality conditions and zero initial conditions on the submartingale solution. On the other hand, finite horizon models, where transversality conditions are not needed, can admit solutions with transient periods of explosive bubbles - see Phillips and Lee (2015a) for this possibility.
} 
encouraging the choice of an upper bound of unity for $\beta$ which raises the convergence rate to the optimal level but invalidates inference.

To fix ideas let $m=1, K=1$ and $\mu=0$ in (4.1). Then

$$
\begin{aligned}
& y_{t+1}=a x_{t}+u_{0 t+1}, \\
& x_{t+1}=\rho x_{t}+u_{x t+1}, \rho=1+\frac{c}{n}, c \in(-\infty, \infty)
\end{aligned}
$$

Our analysis focuses on the (I2)-(I4) cases. Based on the IVX construction 2.9$]$ with $\rho_{z}=1+\frac{c_{z}}{n^{\beta}}$, the generated $\mathrm{AR}(1)$ IVX series is

$$
\tilde{z}_{t+1}=\rho_{z} \tilde{z}_{t}+\triangle x_{t} \sim \rho_{z} \tilde{z}_{t}+u_{x t+1},
$$

and it is straightforward to show the decomposition

$$
\left(\hat{a}_{I V X}-a\right)=\frac{\sum_{t=1}^{n} \tilde{z}_{t} u_{0 . x t+1}}{\sum_{t=1}^{n} \tilde{z}_{t} x_{t}}+\left(\frac{\Sigma_{0 x}}{\Omega_{x x}}\right) \frac{\sum_{t=1}^{n} \tilde{z}_{t} u_{x t+1}}{\sum_{t=1}^{n} \tilde{z}_{t} x_{t}}
$$

where $u_{0 . x t+1}=u_{0 t+1}-\frac{\Sigma_{0 x}}{\Omega_{x x}} u_{x t+1}$ and $\Omega_{x x}$ and $\Sigma_{0 x}$ are the corresponding (long-run) covariances. This decomposition is frequently used in the predictive regression literature and is adapted here to the IVX regression framework to investigate possible choices of $\beta$.

By IVX limit theory we may use

$$
\frac{\sum_{t=1}^{n} \tilde{z}_{t} u_{x t+1}}{\sum_{t=1}^{n} \tilde{z}_{t} x_{t}}=\frac{\sum_{t=1}^{n} \tilde{z}_{t} u_{x t+1}}{\sum_{t=1}^{n} \tilde{z}_{t}^{2}}+o_{p}(1)=\left(\hat{\rho}_{z}-\rho_{z}\right)+o_{p}(1)
$$

since the difference between $\sum_{t=1}^{n} \tilde{z}_{t} x_{t}$ and $\sum_{t=1}^{n} \tilde{z}_{t}^{2}$ is negligible for the (I2)-(I4) cases, as shown in MP. Then

$$
\begin{aligned}
\left(\hat{a}_{I V X}-a\right) & \sim \frac{\sum_{t=1}^{n} \tilde{z}_{t} u_{0 . x t+1}}{\sum_{t=1}^{n} \tilde{z}_{t} x_{t}}+\left(\frac{\Sigma_{0 x}}{\Omega_{x x}}\right)\left(\hat{\rho}_{z}-\rho_{z}\right) \\
& =\frac{\sum_{t=1}^{n} \tilde{z}_{t} u_{0 . x t+1}}{\sum_{t=1}^{n} \tilde{z}_{t} x_{t}}+\left(\frac{\Sigma_{0 x}}{\Omega_{x x}}\right)\left(\hat{\rho}_{z}-\rho_{z}-\frac{1}{n^{\beta}} \operatorname{Bias}\left(\hat{\rho}_{z}\right)\right)+\frac{1}{n^{\beta}}\left(\frac{\Sigma_{0 x}}{\Omega_{x x}}\right) \operatorname{Bias}\left(\hat{\rho}_{z}\right)
\end{aligned}
$$

where the expression for the bias, Bias $\left(\hat{\rho}_{z}\right)$, can be found in Theorem 4.2 in Phillips and Magdalinos (2007b). Next observe that

$n^{\frac{1+\beta}{2}}\left(\hat{a}_{I V X}-a\right)=\frac{n^{-\frac{1+\beta}{2}} \sum_{t=1}^{n} \tilde{z}_{t} u_{0 . x t+1}}{n^{-(1+\beta)} \sum_{t=1}^{n} \tilde{z}_{t} x_{t}}+\left(\frac{\Sigma_{0 x}}{\Omega_{x x}}\right) n^{\frac{1+\beta}{2}}\left(\hat{\rho}_{z}-\rho_{z}-\frac{1}{n^{\beta}} \operatorname{Bias}\left(\hat{\rho}_{z}\right)\right)+n^{\frac{1-\beta}{2}}\left(\frac{\Sigma_{0 x}}{\Omega_{x x}}\right) \operatorname{Bias}\left(\hat{\rho}_{z}\right)$

from which we deduce that

$$
A M S E\left(\hat{a}_{I V X}\right)=\frac{1}{n^{1+\beta}} V+\frac{1}{n^{2 \beta}} B^{2}
$$

where $V=\operatorname{var}\left(\hat{a}_{I V X}\right)$ and $B=\operatorname{bias}\left(\hat{a}_{I V X}\right)$ symbolically. Since $A M S E\left(\hat{a}_{I V X}\right)$ is strictly decreas- 
ing in $\beta \in(0,1)$ this criterion suggests that $\beta$ be chosen as close to unity as possible. This analytical approach therefore provides no informative guidance on an upper bound $\bar{\beta}<1$ for use in practical work. Simulations with cross-validation methods (not reported here) show that these methods encounter the same difficulty. On the other hand, our own simulation exercises investigating different choices for $\beta$ in IVX regression broadly corroborate findings reported in Kostakis et al (2014) that a choice of $\beta=0.95$ works well in terms of size control and power performance for a wide variety of regressors.

\subsection{Simulation-based guidance}

This section reports simulations performed to assess the performance of IVX and provide some practical guidance on suitable IVX persistence $\left(\beta\right.$ or $\left.R_{n z}\right)$ choices in finite sample sizes. We follow the same DGP as in (4.1) with innovations

$$
u_{t}=\left(\begin{array}{l}
u_{0 t} \\
u_{x t}
\end{array}\right) \sim \operatorname{iid} N(0, \Sigma) .
$$

The IVX instruments are constructed as in (2.9) and inference is based on the IVX estimator (2.17). The intercept in 4.1 is handled by demeaning, as explained in Section 2.5. We set $C_{z}=-5$ and vary $\beta$ to explore size and power properties according to different degrees of IVX persistence. In finite samples of size $n=100,250$, the value $C_{z}=-5$ and various choices of $\beta \in(1 / 2,1$ ) (or $\left.\beta \in\left(\alpha_{2}, 1\right)\right)$ deliver a suitably wide range of autoregressive coefficients $R_{n z}$ for the generation of the IVX instruments for investigation.

\subsubsection{Single regressor}

We first run simulations with a single local to unity regressor (i.e. with localizing exponent $\alpha=1$ ). Although some of the main results in the paper relate to locally and mildly explosive regressors, we include results for stationary-side local to unity and unit root cases as well. Accordingly, localization coefficients for the regressor are selected from $c \in\{-50,-20,-10,-5,0,1,3\}$. The first five cases $\{-50,-25,-5,-2,0\}$ have been commonly studied in earlier works. The explosive choices $\{1,3\}$ are new. The variance matrix of the innovations in 4.4 is parameterized as

$$
\Sigma=\left(\begin{array}{ll}
1 & \delta \\
\delta & 1
\end{array}\right)
$$

with $\delta=-0.95$. This value reflects realistic error correlation in predictive regressions and is commonly used in simulations reported in the literature. Since existing studies typically focus on a single predictor and a one-sided test, we similarly consider here tests of the null $H_{0}: a=0$ against $H_{1}: a>0$.

Figure $15^{5}$ summarizes the size and power performance of IVX tests using $\beta=0.95$, and includes

\footnotetext{
${ }^{5}$ This Figure 1 is comparable to Figure 1 of Kostakis et al. (2014), although we report results for a one sided test
} 
the Campbell-Yogo Q (CY-Q) test and its modified version for comparison ${ }^{6}$. In view of the extensive simulation results reported in Kostakis et al. (2014), we include a few representative cases here that highlight some of the new aspects of the limit theory.

- Figure 1 Here -

The invalidity of the CY-Q test for stationary and certain mildly integrated regressors was demonstrated recently in Phillips (2014) and that failure in this test is clearly evident in the first two graphs of Figure $1(c=-50, c=-25$, with $n=250)$ where CY-Q has zero size and low power. It is therefore particularly interesting to see the performance of the modified version of the CY-Q test that avoids the centering difficulties of the CY-Q test. In fact, the modified version of the CY-Q test shows good performance for these cases but its power function is dominated by IVX power for these cases and for all other values of $c$ except $c=0$. The modified CY-Q test performs well in the unit root case in terms of both size control and power. Overall, the results show greater reliability for the IVX test than either version of the two CY-Q tests.

These results corroborate the recommendation made in Kostakis et al. (2014) for use of the parameter setting $\beta=0.95$ in empirical practice with IVX. The results here support the use of this choice for a range of empirically relevant persistent regressors in the $\mathrm{I}(1)-\mathrm{I}(5)$ class.

\subsubsection{Multiple regressors}

We next consider the empirically relevant case of multiple predictive regressors. We take the bivariate case $K=2$ in (4.1) to illustrate and consider four scenarios: (i) stationary local to unity and unit root cases $\left(c_{1}=-25\right.$ or -5 and $c_{2}=0$ ); (ii) bivariate unit root predictors $\left(c_{1}=c_{2}=0\right.$ ), (iii) stationary local to unity and mildly explosive root cases $\left(c_{1}=-25\right.$ or -5 and $\left.c_{2}=3\right)$; and (iv) unit root and mildy explosive root case $\left(c_{1}=0\right.$ and $\left.c_{2}=3\right)$. These scenarios mimic several common empirical situations, including the practical example considered later of exchange rate predictive regression with two persistent predictors. The innovation structure follows (4.4) and uses the specific correlation matrix estimated in the empirical application in Section 57

$$
\Sigma=\left(\begin{array}{ccc}
1 & 0.10 & -0.29 \\
0.10 & 1 & -0.03 \\
-0.29 & -0.03 & 1
\end{array}\right) \text {. }
$$

Figure 2 reports size and power performance in testing the joint predictive regression hypothesis $H_{0}: a_{1}=a_{2}=0$ using the $\chi^{2}$ test of Theorem 3.2 and the same demeaning procedure from Section

while Kostakis et al. use a two sided test. Comparisons are given for explosive cases with $c=1,3$ for CY-Q test, in view of the limited availability of critical values for the Modified CY-Q test.

${ }^{6}$ The modified CY-Q test uses a uniformly valid confidence interval (CI) for $\rho$, rather than Stock's CI (1991) to avoid the invalidity issue with stationary predictor. In particular, we use the critical values from Andrews and Guggenberger (2014, Table 2).

${ }^{7}$ We selectively report here results with bivariate predictors that mimic the scenario in the empirical section below. Other scenarios considered in the simulations are available upon request. 
2.5. The IVX persistence parameter is selected from $\beta \in\{0.85,0.90,0.95\}$ and the localizing coefficients are set to $C_{z}=\operatorname{diag}(-5,-5)$. The nominal (asymptotic) test size is 0.05 , the sample size $n=100$, and the number of replications is 2,500. Again, finite sample test size is evidently well controlled, with increasing local power produced as $\beta$ increases. Local power reaches unity quickly for the mixed root cases with a mildly explosive regressor.

- Figure 2 Here -

These results reveal that finite sample size and power of IVX predictability tests seem reliable across a range of different cases. Flexible choices of $\beta \in\{0.85,0.90,0.95\}$ with $C_{z}=-5$ seem to work well for finite sample sizes as low as $n=100$, even for multiple regressor cases, and for regressors in classes (I1)-(I6). The findings generally corroborate those of Kostakis et al (2014).

\section{Conclusion}

This paper shows that the IVX method of Magdalinos and Phillips (2009b) is robust under locally and mildly explosive regressors as well as certain combinations of mixed integrated regressors. The models we have dealt with in the present paper are prototypical with features that allow for mixed regressors, giving a limit theory that shows the easy amenability of IVX methodology to standard testing procedures. However, while suggestive, these models do not provide a fully general environment that admits multiple regressors with variable localizing exponents, either for the mildly integrated or the mildly explosive regressors. Similarly, it does not provide a general limit theory of Wald testing with arbitrary restrictions for mixed regressors. Analytic research on the properties of IVX in such general environments is ongoing work by Magdalinos and Phillips. But the partial results provided here, combined with those in Kostakis et al (2014) are highly suggestive of the potential of these method to deal with mixed degrees of persistence, uncertainty over persistence degrees, and multiple regressors with different time series memory characteristics and long run properties.

A limitation of the IVX approach is that the localizing parameter $\beta$ used in the construction of the instruments must be chosen by the empirical investigator. Asymptotic theory justifies a wide range of flexibility in the choice of $\beta$ provided some general restrictions on this parameters are observed. But since the localizing rate $\alpha$ that controls the degree of persistence in the regressors is unknown these restrictions are imperfectly known. A convenient approach for much practical work is for investigators to simply assume that the regressors have (local) unit roots, or mildly explosive. This framework covers most practical situations including those that are commonly used in predictive regression, giving an assumed rate of $\alpha=1$ for the regressors. For this general setting, theory indicates that the procedure offers a wide degree of flexibility in the choice of $\beta$ and the construction of IVX instruments. Simulations confirm that this flexibility continues to hold in finite samples and that good size and power properties hold for various choices of $\beta \in[0.85,0.95]$, with $\beta=0.95$ typically producing greatest discriminatory power while still controlling size. 
Figure 1: Empirical size/power performances of IVX, CY-Q and Modified CY-Q tests
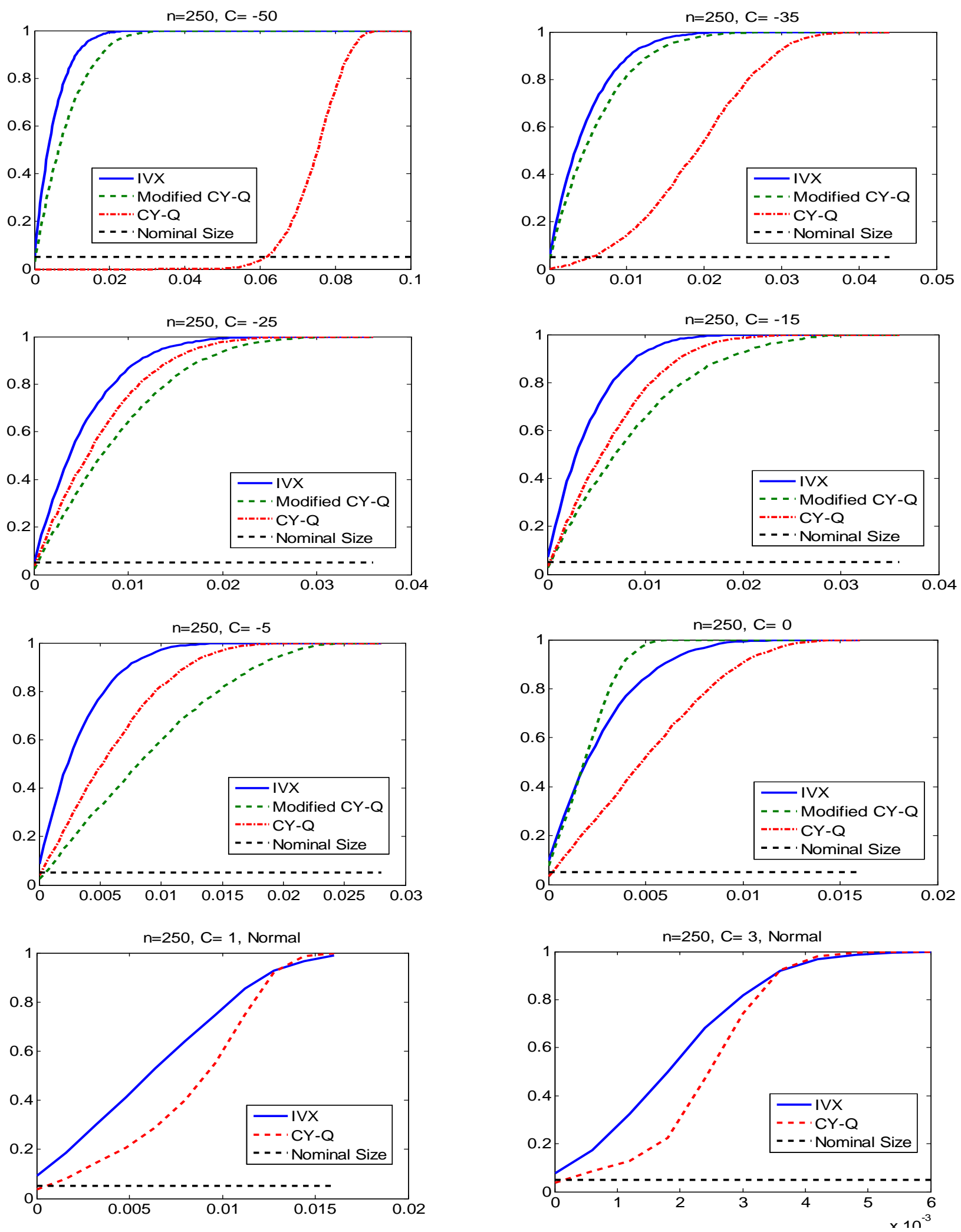
Figure 2: Empirical size/power of IVX tests with bivariate regressors
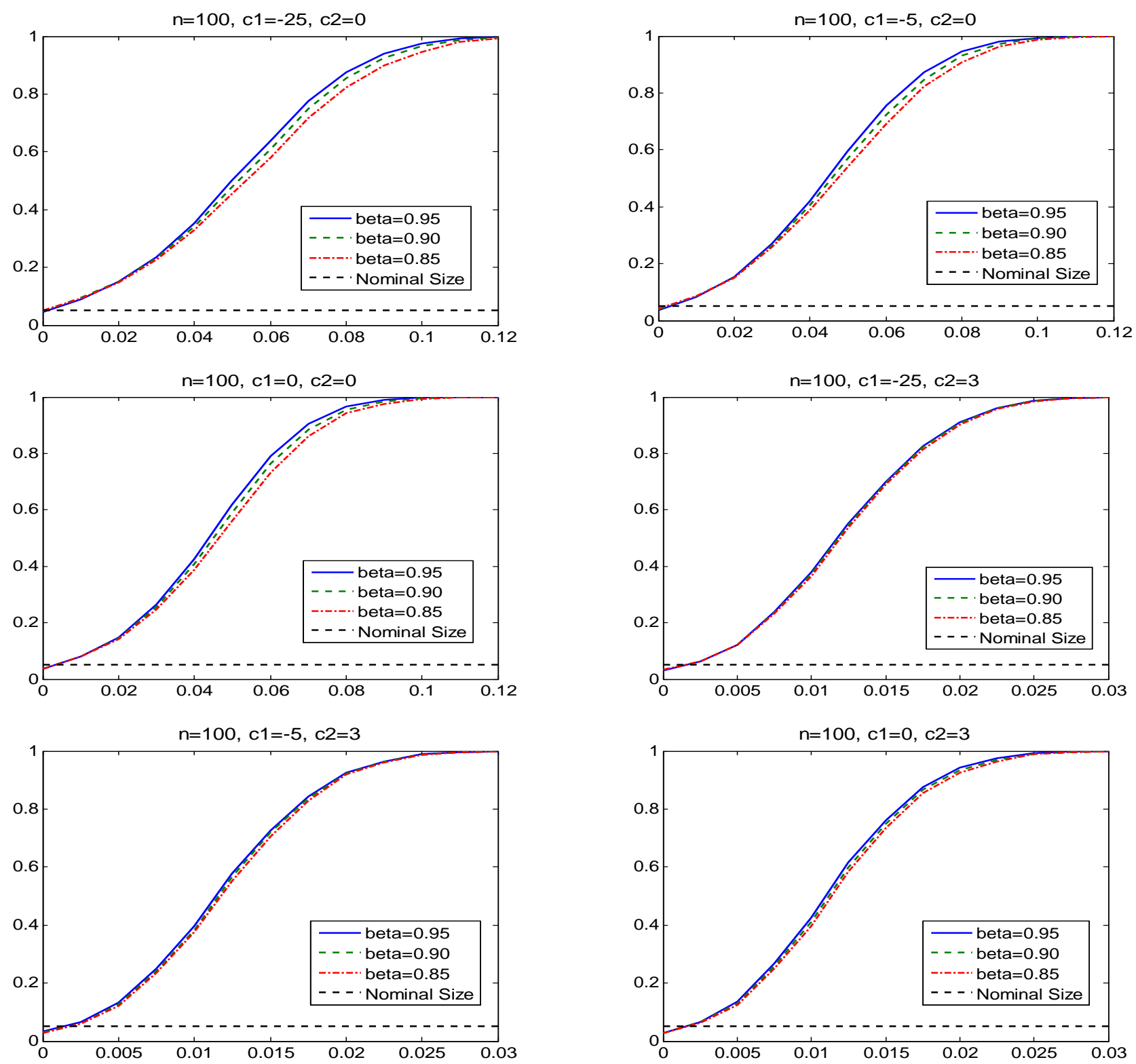

\section{Technical Appendix}

This Appendix provides some useful preliminary lemmas and their proofs as well as proofs of the main theorems in the paper. The LUR explosive and mildly explosive cases are investigated in the following sections and main proofs for the case of mixed roots follow. 


\subsection{LUR explosive Regressors: (I4)}

We consider the (I4) LUR explosive case $(C>0, \alpha=1)$ with $\frac{1}{2}<\beta<1$. Here, the same limit theory as MP continues to hold.

Lemma 6.1 (Lemma 3.1 in PM with $C>0$ and $\alpha=1$ )

1. $n^{-\frac{1+\beta}{2}} \sum_{t=1}^{n} u_{0 t} \tilde{z}_{t}^{\prime}=n^{-\frac{1+\beta}{2}} \sum_{t=1}^{n} u_{0 t} z_{t}^{\prime}+o_{p}(1)$

2. $n^{-(1+\beta)} \sum_{t=1}^{n} x_{t} \tilde{z}_{t}^{\prime}=n^{-(1+\beta)} \sum_{t=1}^{n} x_{t} z_{t}^{\prime}-n^{-2} \sum_{t=1}^{n} x_{t-1} x_{t-1}^{\prime} C C_{z}^{-1}+o_{p}(1)$

3. $n^{-(1+\beta)} \sum_{t=1}^{n} \tilde{z}_{t} \tilde{z}_{t}^{\prime}=n^{-(1+\beta)} \sum_{t=1}^{n} z_{t} z_{t}^{\prime}+o_{p}(1)$

Proof. 1. Propositions A1 and A2 in MP hold with $C>0$ and $\alpha=1$ without any substantial change in the proofs. To get the uniform bound for $E\left\|\psi_{n t}\right\|^{2}$, instead of using $\left\|R_{n}\right\|^{i-l} \leq 1$ for $l \leq i$ when $C<0$, we can use when $C>0$,

$$
\left\|R_{n}\right\|^{i-l} \leq \exp (C)+o(1), \text { for } i-l \leq n,
$$

and we still have the same order of magnitude $\sup _{1 \leq t \leq n} E\left\|\psi_{n t}\right\|^{2}=O\left(n^{1+2 \beta}\right)$ implying

$$
n^{-\left(\frac{1+\beta}{2}+1\right)} \sum_{t=1}^{n} u_{0 t} \psi_{n t}^{\prime}=o_{p}(1), \text { and } n^{-\frac{1+\beta}{2}} \sum_{t=1}^{n} u_{0 t} \tilde{z}_{t}^{\prime}=n^{-\frac{1+\beta}{2}} \sum_{t=1}^{n} u_{0 t} z_{t}^{\prime}+o_{p}(1) .
$$

Consequently, the limit theory of the numerator $n^{-\frac{1+\beta}{2}} \sum_{t=1}^{n} u_{0 t} \tilde{z}_{t}^{\prime}$ does not involve $C$ at all. The proofs of 2 and 3 do not depend on the sign of $C$ and use the distributional limit result

$$
n^{-1 / 2} x_{\lfloor n s\rfloor} \Longrightarrow J_{x}^{c}(r)
$$

for $C>0$ from Phillips (1987).

The limit theory of the IVX estimator (2.11) is therefore the same as the case under (I2), or $\mathrm{N}$ (ii) in MP, proving Theorem 3.1-1 and 3.2 for (I4) LUR explosive regressors.

\subsection{Mildly Explosive Regressors: (I5)}

We collect proofs for the (I5) mildly explosive regressor case here. As in MP, we consider two possible cases $\frac{2}{q}<\beta<\alpha<1$ and $\frac{2}{q}<\alpha<\beta<1$. In both cases, IVX asymptotics with mildly explosive regressors is developed and the pivotal chi-square limit theory is shown to be valid.

Proof of Lemma 2.2 (LP case). By back substitution and from the linear BN decomposition of $u_{t}$,

$$
\frac{1}{n^{\alpha / 2}} R_{n}^{-(j-1)} x_{j-1}=\frac{1}{n^{\alpha / 2}} \sum_{i=1}^{j-1} R_{n}^{-i} u_{x i}=\frac{1}{n^{\alpha / 2}} \sum_{i=1}^{j-1} R_{n}^{-i} F_{x}(1) \varepsilon_{i}-\frac{1}{n^{\alpha / 2}} \sum_{i=1}^{j-1} R_{n}^{-i} \triangle \tilde{\varepsilon}_{x i}, \quad \text { under LP . }
$$


Consider the partial sum process $\eta_{n}(r)=\frac{1}{\sqrt{n}} S_{\lfloor n r\rfloor}$ with $S_{j}=\sum_{i=1}^{j} \varepsilon_{i}$. Using a strong approximation, we can enlarge the original probability space and construct a vector Brownian motion $\omega=B M(\Sigma)$ on this space with the property that for $\varepsilon>0, E\left[\left\|\varepsilon_{i}\right\|^{q}\right]<\infty$,

$$
\sup _{0 \leq r \leq 1}\left\|\eta_{n}(r)-\omega(r)\right\|=o_{a . s .}\left(n^{-1 / 2+1 / q+\varepsilon}\right) .
$$

Define $\frac{e_{i}}{\sqrt{n}}=\omega\left(\frac{i}{n}\right)-\omega\left(\frac{i-1}{n}\right)$ whose distribution is iid normal with $E\left(e_{i} e_{i}^{\prime}\right)=\Sigma$. We generate another mildly explosive $\operatorname{AR}(1)$ process, $z_{i}=R_{n} z_{i-1}+v_{x i}$, where, under LP, $v_{x i}=F_{x}(1) e_{i}-\triangle \widetilde{e}_{x i}$ is a linear process defined in a similar way to $u_{x i}$ but using $e_{i}$ instead of $\varepsilon_{i}$. Then

$$
\frac{1}{n^{\alpha / 2}} R_{n}^{-(j-1)} z_{j-1}=\frac{1}{n^{\alpha / 2}} \sum_{i=1}^{j-1} R_{n}^{-i} F_{x}(1) e_{i}-\frac{1}{n^{\alpha / 2}} \sum_{i=1}^{j-1} R_{n}^{-i} \triangle \widetilde{e}_{x i}, \quad \text { under LP . }
$$

Letting $j_{n}=j-1$ be such that $k_{n} \leq j_{n} \leq n$ we have, under LP,

$$
\frac{1}{n^{\alpha / 2}} \sum_{i=1}^{j_{n}} R_{n}^{-i} F_{x}(1) e_{i}:=\tilde{Y}_{C n, j_{n}} \equiv N\left(0, \frac{1}{n^{\alpha}} \sum_{i=1}^{j_{n}} R_{n}^{-i} \Omega_{x x} R_{n}^{-i}\right) \text {, }
$$

by construction. Define the limit process $\lim _{n} \tilde{Y}_{C n, j_{n}}=\tilde{Y}_{C} \equiv N\left(0, \int_{0}^{\infty} e^{-p C} \Omega_{x x} e^{-p C} d p\right)$. The strong approximation between these two processes will be shown below in (iii). Observe that

$$
\begin{aligned}
& \left\|\frac{1}{n^{\alpha / 2}} R_{n}^{-(j-1)} x_{j_{n}}-\tilde{Y}_{C}\right\| \leq\left\|\frac{1}{n^{\alpha / 2}} \sum_{i=1}^{j-1} R_{n}^{-i} F_{x}(1) \varepsilon_{i}-\frac{1}{n^{\alpha / 2}} \sum_{i=1}^{j-1} R_{n}^{-i} F_{x}(1) e_{i}\right\| \\
& +\left\|\tilde{Y}_{C n}-\tilde{Y}_{C}\right\|+\left\|\frac{1}{n^{\alpha / 2}} \sum_{i=1}^{j-1} R_{n}^{-i} \triangle \tilde{\varepsilon}_{x i}\right\|+\left\|\frac{1}{n^{\alpha / 2}} \sum_{i=1}^{j-1} R_{n}^{-i} \triangle \widetilde{e}_{x i}\right\| .
\end{aligned}
$$

We now show that each term in (6.1) is $o_{a . s .}(1)$ uniformly over $j-1=j_{n} \in\left[k_{n}, n\right]$.

(i) $\sup _{k_{n} \leq j-1 \leq n}\left\|\frac{1}{n^{\alpha / 2}} \sum_{i=1}^{j-1} R_{n}^{-i} F_{x}(1) \varepsilon_{i}-\frac{1}{n^{\alpha / 2}} \sum_{i=1}^{j-1} R_{n}^{-i} F_{x}(1) e_{i}\right\|=o_{a . s}(1)$.

$$
\begin{gathered}
\frac{1}{n^{\alpha / 2}} \sum_{i=1}^{j-1} R_{n}^{-i} F_{x}(1) \varepsilon_{i}=\frac{n^{1 / 2}}{n^{\alpha / 2}} \sum_{i=1}^{j-1} R_{n}^{-i} F_{x}(1)\left(\eta_{n}\left(\frac{i}{n}\right)-\eta_{n}\left(\frac{i-1}{n}\right)\right) \\
=\frac{n^{1 / 2}}{n^{\alpha / 2}}\left\{\sum_{i=1}^{j-1} R_{n}^{-i} F_{x}(1) \eta_{n}\left(\frac{i}{n}\right)-\sum_{i=1}^{j-1} R_{n}^{-i} F_{x}(1) \eta_{n}\left(\frac{i-1}{n}\right)\right\} \\
=\frac{n^{1 / 2}}{n^{\alpha / 2}}\left\{\sum_{i=1}^{j-1} R_{n}^{-i} F_{x}(1) \eta_{n}\left(\frac{i}{n}\right)-\sum_{s=0}^{j-2} R_{n}^{-s-1} F_{x}(1) \eta_{n}\left(\frac{s}{n}\right)\right\} \\
=\frac{n^{1 / 2}}{n^{\alpha / 2}}\left\{\sum_{i=1}^{j-1} R_{n}^{-i} F_{x}(1) \eta_{n}\left(\frac{i}{n}\right)-\sum_{i=1}^{j-1} R_{n}^{-i-1} F_{x}(1) \eta_{n}\left(\frac{i}{n}\right)+R_{n}^{-j-2} F_{x}(1) \eta_{n}\left(\frac{j-1}{n}\right)\right\} \\
=\frac{n^{1 / 2}}{n^{\alpha / 2}}\left\{\left(1-R_{n}^{-1}\right) \sum_{i=1}^{j-1} R_{n}^{-i} F_{x}(1) \eta_{n}\left(\frac{i}{n}\right)+R_{n}^{-j-2} F_{x}(1) \eta_{n}\left(\frac{j-1}{n}\right)\right\} .
\end{gathered}
$$


Similarly,

$$
\frac{1}{n^{\alpha / 2}} \sum_{i=1}^{j-1} R_{n}^{-i} F_{x}(1) e_{i}=\frac{n^{1 / 2}}{n^{\alpha / 2}}\left\{\left(1-R_{n}^{-1}\right) \sum_{i=1}^{j-1} R_{n}^{-i} F_{x}(1) \omega\left(\frac{i}{n}\right)+R_{n}^{-j-2} F_{x}(1) \omega\left(\frac{j-1}{n}\right)\right\} .
$$

Therefore,

$$
\begin{aligned}
& \left\|\frac{1}{n^{\alpha / 2}} \sum_{i=1}^{j-1} R_{n}^{-i} F_{x}(1) \varepsilon_{i}-\frac{1}{n^{\alpha / 2}} \sum_{i=1}^{j-1} R_{n}^{-i} F_{x}(1) e_{i}\right\| \\
\leq & \frac{n^{1 / 2}}{n^{\alpha / 2}}\left\|F_{x}(1)\right\|\left(\sup _{0 \leq r \leq 1}\left\|\eta_{n}(r)-\omega(r)\right\|\right)\left\|1-R_{n}^{-1}\right\| \sum_{i=1}^{j-1}\left\|R_{n}\right\|^{-i} \\
& +\frac{n^{1 / 2}}{n^{\alpha / 2}}\left\|R_{n}\right\|^{-j-2}\left\|F_{x}(1)\right\|\left(\sup _{0 \leq r \leq 1}\left\|\eta_{n}(r)-\omega(r)\right\|\right) \\
= & \frac{n^{1 / 2}}{n^{\alpha / 2}}\left(\sup _{0 \leq r \leq 1}\left\|\eta_{n}(r)-\omega(r)\right\|\right)\left\|F_{x}(1)\right\|\left(\left\|1-R_{n}^{-1}\right\| \sum_{i=1}^{j-1}\left\|R_{n}\right\|^{-i}+\left\|R_{n}\right\|^{-j-2}\right),
\end{aligned}
$$

and for $k_{n} \leq j \leq n,\left(\left\|1-R_{n}^{-1}\right\| \sum_{i=1}^{j-1}\left\|R_{n}\right\|^{-i}+\left\|R_{n}\right\|^{-j-2}\right)$ is $O(1)$ since $\left\|R_{n}\right\|^{-j} \leq \exp \left(-\min \left(c_{i}\right) \frac{j}{n^{\alpha}}\right)+$ $o(1)=o(1)$ with $\frac{n^{\alpha}}{k_{n}}+\frac{n^{\alpha}}{n} \rightarrow 0$.

Therefore,

$\sup _{k_{n} \leq j-1 \leq n}\left\|\frac{1}{n^{\alpha / 2}} \sum_{i=1}^{j-1} R_{n}^{-i} F_{x}(1) \varepsilon_{i}-\frac{1}{n^{\alpha / 2}} \sum_{i=1}^{j-1} R_{n}^{-i} F_{x}(1) e_{i}\right\|=\frac{n^{1 / 2}}{n^{\alpha / 2}} o_{a . s .}\left(n^{-1 / 2+1 / q+\varepsilon}\right)=o_{a . s .}\left(n^{\frac{1}{q}-\frac{\alpha}{2}+\varepsilon}\right)$,

and the exponent $\frac{1}{q}-\frac{\alpha}{2}+\varepsilon<0$ for small enough $\varepsilon$ because $\alpha>\frac{2}{q}$ and $q \geq 4$. The strong approximation is therefore sharper under higher moment conditions and when the signal strength of the mildly explosive regressors is closer to the local to unity region. Conversely, if the signal strength is closer to the purely explosive case $\left(\alpha \leq \frac{1}{2}\right)$ higher moment conditions are needed to ensure that $\frac{1}{q}-\frac{\alpha}{2}+\varepsilon<0$. The approximation is not possible in the pure explosive case $(\alpha=0)$.

(ii) $\sup _{k_{n} \leq j-1 \leq n}\left\|\frac{1}{n^{\alpha / 2}} \sum_{i=1}^{j-1} R_{n}^{-i} \triangle \tilde{\varepsilon}_{x i}\right\|=o_{a . s}(1)=\sup _{k_{n} \leq j-1 \leq n}\left\|\frac{1}{n^{\alpha / 2}} \sum_{i=1}^{j-1} R_{n}^{-i} \triangle \widetilde{e}_{x i}\right\|$.

Using summation by parts

$$
\frac{1}{n^{\alpha / 2}} \sum_{i=1}^{j-1} R_{n}^{-i} \triangle \tilde{\varepsilon}_{x i}=-\frac{1}{n^{\alpha / 2}} \sum_{i=1}^{j-2} \triangle R_{n}^{-i-1} \tilde{\varepsilon}_{x i}-\frac{R_{n}^{-1}}{n^{\alpha / 2}} \tilde{\varepsilon}_{x 0}+\frac{R_{n}^{-(j-1)}}{n^{\alpha / 2}} \tilde{\varepsilon}_{x j-1},
$$

and from the given moment condition

$$
\begin{aligned}
& P\left(\left\|\frac{1}{n^{\alpha / 2}} \tilde{\varepsilon}_{x n}\right\|>\epsilon\right) \leq \frac{E\left\|\tilde{\varepsilon}_{x n}\right\|^{q}}{n^{\frac{q \alpha}{2}} \epsilon^{q}}=O\left(\frac{1}{n^{\frac{q \alpha}{2}}}\right), \\
& \sum_{n=1}^{\infty} P\left(\left\|\frac{1}{n^{\alpha / 2}} \tilde{\varepsilon}_{x n}\right\|\right) \leq O\left(\sum_{n=1}^{\infty} \frac{1}{n^{\frac{q \alpha}{2}}}\right)<\infty,
\end{aligned}
$$


as long as $q \alpha>2$ which is satisfied by the condition $\alpha>\frac{2}{q}$. Thus, $n^{-\alpha / 2} R_{n}^{-1} \tilde{\varepsilon}_{x 0}=o_{a . s .}$. 1 ) by the Borel-Cantelli lemma. Using the fact that with $\left\|R_{n}\right\|^{-j}=o(1)$ when $j-1 \in\left[k_{n}, n\right]$, we also have $n^{-\alpha / 2} R_{n}^{-(j-1)} \tilde{\varepsilon}_{x j-1}=o_{a . s .}(1)$. Note further that

$$
\frac{1}{n^{\alpha / 2}} \sum_{i=1}^{j-2} \triangle R_{n}^{-i-1} \tilde{\varepsilon}_{x i}=\frac{R_{n}^{-1} C}{n^{\frac{3 \alpha}{2}}} \sum_{i=1}^{j-2} R_{n}^{-i} \tilde{\varepsilon}_{x i}
$$

and $R_{n}^{-1} C=O(1)$. For $k_{n} \leq j \leq n$

$$
E\left(\left\|\frac{1}{n^{\frac{3 \alpha}{2}}} \sum_{i=1}^{j-2} R_{n}^{-i} \tilde{\varepsilon}_{x i}\right\|^{q}\right)^{\frac{1}{q}}=\left\|\frac{1}{n^{\frac{3 \alpha}{2}}} \sum_{i=1}^{j-2} R_{n}^{-i} \tilde{\varepsilon}_{x i}\right\|_{L_{q}} \leq \frac{\left\|\tilde{\varepsilon}_{x i}\right\|_{L_{q}}}{n^{\frac{3 \alpha}{2}}} \sum_{i=1}^{j-2}\left\|R_{n}\right\|^{-i}=O\left(\frac{1}{n^{\frac{\alpha}{2}}}\right),
$$

since $\tilde{\varepsilon}_{x i}$ is stationary and $n^{-\alpha} \sum_{i=1}^{j-2}\left\|R_{n}\right\|^{-i}=O(1)$ for $k_{n} \leq j \leq n$. Hence

$$
E\left(\left\|\frac{1}{n^{\frac{3 \alpha}{2}}} \sum_{i=1}^{j-2} R_{n}^{-i} \widetilde{\varepsilon}_{x i}\right\|^{q}\right)=O\left(\frac{1}{n^{\frac{q \alpha}{2}}}\right)
$$

and

$$
P\left(\left\|\frac{1}{n^{\frac{3 \alpha}{2}}} \sum_{i=1}^{j-2} R_{n}^{-i} \tilde{\varepsilon}_{x i}\right\|>\epsilon\right) \leq \frac{E\left(\left\|\frac{1}{n^{\frac{3 \alpha}{2}}} \sum_{i=1}^{j-2} R_{n}^{-i} \tilde{\varepsilon}_{x i}\right\|^{q}\right)}{\epsilon^{\frac{q}{2}}}=O\left(\frac{1}{n^{\frac{q \alpha}{2}}}\right) .
$$

We then have $n^{-3 \alpha / 2} R_{n}^{-1} C \sum_{i=1}^{j-2} R_{n}^{-i} \widetilde{\varepsilon}_{x i}=o_{a . s}(1)$ under the given moment conditions just as before. The proof for $n^{-\alpha / 2} \sum_{i=1}^{j-1} R_{n}^{-i} \triangle \widetilde{e}_{x i}$ is exactly same.

(iii) $\left\|\tilde{Y}_{C n}-\tilde{Y}_{C}\right\|=o_{a . s .}(1)$

For clarity, we denote $n^{-\alpha / 2} \sum_{i=1}^{j-1} R_{n}^{-i} F_{x}(1) e_{i}=\tilde{Y}_{C n}:=\tilde{Y}_{C n, j-1}$, and define the martingale array $\left\{\mathcal{F}_{n, j-1}, \tilde{Y}_{C n, j-1}: j-1 \geq k_{n}\right\}$ with natural filtration $\mathcal{F}_{n, j-1}$. We have $E_{\mathcal{F}_{n, j-1}}\left[\tilde{Y}_{C n, j}\right]=\tilde{Y}_{C n, j-1}$ and

$$
E\left[\tilde{Y}_{C n, j} \tilde{Y}_{C n, j}^{\prime}\right]=\frac{1}{n^{\alpha}} \sum_{i=1}^{j-1} R_{n}^{-i} \Omega_{x x} R_{n}^{-i} \rightarrow \int_{0}^{\infty} e^{-p C} \Omega_{x x} e^{-p C} d p<\infty \text { for } j-1 \geq k_{n} .
$$

By the martingale convergence theorem for $L_{2}$-bounded martingales (e.g., Hall and Heyde, 1980), $\tilde{Y}_{C n, j-1} \rightarrow a . s . \tilde{Y}_{C} \equiv N\left(0, \int_{0}^{\infty} e^{-p C} \Omega_{x x} e^{-p C} d p\right)$, which is a distributionally equivalent copy of $Y_{C}$. Combining (i)-(iii) gives the required result.

Proof of Lemma 2.2 (NLP). From the nonlinear BN decomposition of $u_{t}$ we have

$$
\begin{aligned}
\frac{1}{n^{\alpha / 2}} R_{n}^{-(j-1)} x_{j-1} & =\frac{1}{n^{\alpha / 2}} \sum_{i=1}^{j-1} R_{n}^{-i} u_{x i} \\
& =\frac{1}{n^{\alpha / 2}} \sum_{i=1}^{j-1} R_{n}^{-i} D_{x i}-\frac{1}{n^{\alpha / 2}} \sum_{i=1}^{j-1} R_{n}^{-i} \triangle \tilde{D}_{x i}, \quad \text { under NLP . }
\end{aligned}
$$

We modify the proof of Theorem 3 in Wu (2007), where a strong invariance principle for a nonweighted partial sum $\left(\sum_{i} u_{x i}\right)$ was given. The conditions in Theorem 3 of Wu (2007) are met under 
the GMC condition imposed in Assumption NLP. We proceed in two steps. (i) First, we show the remainder term is $o_{a . s .}(1)$ in 6.2 .

$$
\sup _{j_{n}}\left\|\frac{1}{n^{\alpha / 2}} \sum_{i=1}^{j_{n}} R_{n}^{-i} \triangle \tilde{D}_{x i}\right\| \leq \sup _{i \leq j_{n}}\left\|\frac{R_{n}^{-i}}{n^{\alpha / 2}}\right\| \sup _{j_{n}}\left\|\sum_{i=1}^{j_{n}} \triangle \tilde{D}_{x i}\right\|=\frac{1}{n^{\alpha / 2}} \sup _{j_{n}}\left\|\sum_{i=1}^{j_{n}} \triangle \tilde{D}_{x i}\right\|,
$$

and from Theorem 3-(ii) in Wu (2007), it is known that

$$
\sup _{j_{n}}\left\|\sum_{i=1}^{j_{n}} \triangle \tilde{D}_{x i}\right\|=o_{a . s .}\left(n^{1 / q}(\log n)^{1 / 2+1 / q}(\log \log )^{2 / q}\right),
$$

so that

$$
\sup _{j_{n}}\left\|\frac{1}{n^{\alpha / 2}} \sum_{i=1}^{j_{n}} R_{n}^{-i} \triangle \tilde{D}_{x i}\right\|=o_{a . s .}\left(n^{\frac{1}{q}-\frac{\alpha}{2}}(\log n)^{1 / 2+1 / q}(\log \log )^{2 / q}\right)=o_{a . s .}(1)
$$

when $\frac{1}{q}-\frac{\alpha}{2}<0$, as in the proof of LP case. (ii) The strong approximation now follows from

$$
\sup _{j_{n}}\left\|\frac{1}{n^{\alpha / 2}} \sum_{i=1}^{j_{n}} R_{n}^{-i} u_{x i}-\frac{1}{n^{\alpha / 2}} \sum_{i=1}^{j_{n}} R_{n}^{-i} D_{x i}\right\|=o_{a . s .}(1)
$$

by a similar argument, viz.,

$$
\begin{gathered}
\sup _{j_{n}}\left\|\frac{1}{n^{\alpha / 2}} \sum_{i=1}^{j_{n}} R_{n}^{-i} D_{x i}-\tilde{Y}_{C}\right\| \leq \frac{1}{n^{\alpha / 2}} \sup _{j_{n} \leq n}\left\|B_{x}\left(T_{j_{n}}\right)-\omega\left(j_{n}\right)\right\| \\
=o_{a . s .}\left(n^{\frac{1}{q}-\frac{\alpha}{2}}(\log n)^{1 / 2+1 / q}(\log \log )^{2 / q}\right)=o_{a . s .}(1),
\end{gathered}
$$

in which we enlarge the original probability space and construct a vector Brownian motion $\omega=$ $B M\left(\Omega_{x x}\right)$ and partial sums $T_{j_{n}}=\sum_{i=1}^{j_{n}} \tau_{i}$ that have the same conditional mean and variance with $\sum_{i=1}^{j_{n}} D_{x i}$ almost surely (see Hall and Heyde (1980) or page 2314 of $\mathrm{Wu}(2007)$ ). As in LP case, the strong approximation rate is sharper under higher moment conditions and when the signal strength of the mildly explosive regressors is closer to the local to unity region. Higher moment conditions are also needed when the signal strength is closer to the purely explosive case $\left(\alpha \leq \frac{1}{2}\right)$.

Proof of Lemma 2.3. Since $k_{n}$ satisfies $\frac{n^{\alpha \vee \beta}}{k_{n}} \rightarrow 0$, we have both $\left\|R_{n}\right\|^{-k_{n}} \rightarrow 0$ and $\left\|R_{n z}\right\|^{k_{n}} \rightarrow 0$, so the condition for $k_{n}$ in lemma 2.2 holds. In addition $\left\|R_{n z}\right\|^{t} \rightarrow 0$ and $\left\|R_{n}\right\|^{-t} \rightarrow 0$ since $t>k_{n}$. We have

$$
\frac{1}{n^{\frac{\alpha}{2}+\alpha \wedge \beta}} R_{n}^{-t} \psi_{n t}=\frac{1}{n^{\frac{\alpha}{2}+\alpha \wedge \beta}} R_{n}^{-t} \sum_{j=1}^{t} R_{n z}^{t-j} x_{j-1}=\frac{1}{n^{\frac{\alpha}{2}+\alpha \wedge \beta}} R_{n}^{-t} \sum_{j=k_{n}+1}^{t} R_{n z}^{t-j} x_{j-1}+o_{p}(1),
$$

because the frontal summation over $1 \leq j \leq k_{n}$ is negligible as we now show. In particular, using 
(2.13), we have

$$
\begin{gathered}
\left\|\frac{1}{n^{\frac{\alpha}{2}+\alpha \wedge \beta}} R_{n}^{-t} \sum_{j=1}^{k_{n}} R_{n z}^{t-j} x_{j-1}\right\|_{L_{1}}=\left\|\frac{1}{n^{\alpha \wedge \beta}} R_{n}^{-t} \sum_{j=1}^{k_{n}} R_{n z}^{t-j} R_{n}^{(j-1)}\left(\frac{R_{n}^{-(j-1)} x_{j-1}}{n^{\alpha / 2}}\right)\right\|_{L_{1}} \\
\leq \sup _{j-1}\left\|\frac{R_{n}^{-(j-1)} x_{j-1}}{n^{\alpha / 2}}\right\|_{L_{2}} \frac{1}{n^{\alpha \wedge \beta}}\left\|R_{n}\right\|^{-t}\left\|R_{n z}\right\|^{t} \sum_{j=1}^{k_{n}}\left\|R_{n z}\right\|^{-j}\left\|R_{n}\right\|^{j-1} \\
=O\left(\frac{1}{n^{\alpha \wedge \beta}}\left\|R_{n z}\right\|^{t-1}\left\|R_{n}\right\|^{-t} \frac{\left\|R_{n z}\right\|^{-k_{n}}\left\|R_{n}\right\|^{k_{n}}-1}{\left\|R_{n z}\right\|^{-1}\left\|R_{n}\right\|-1}\right) \\
=O\left(\frac{\left\|R_{n z}\right\|^{t-k_{n}-1}\left\|R_{n}\right\|^{k_{n}-t}-\left\|R_{n}\right\|^{-t}\left\|R_{n z}\right\|^{t-1}}{n^{\alpha \wedge \beta}\left(\left\|R_{n z}\right\|^{-1}\left\|R_{n}\right\|-1\right)}\right)=o(1),
\end{gathered}
$$

since

$$
\begin{aligned}
n^{\alpha \wedge \beta}\left(\left\|R_{n z}\right\|^{-1}\left\|R_{n}\right\|-1\right) & =n^{\alpha \wedge \beta}\left(\frac{1+\frac{\max \left(c_{i}\right)}{n^{\alpha}}}{1+\frac{\max \left(c_{z i}\right)}{n^{\beta}}}-1\right)=n^{\alpha \wedge \beta}\left(\frac{\frac{\max \left(c_{i}\right)}{n^{\alpha}}-\frac{\max \left(c_{z i}\right)}{n^{\beta}}}{1+\frac{\max \left(c_{z i}\right)}{n^{\beta}}}\right) \\
& \rightarrow\left\{\begin{array}{cl}
-\max \left(c_{z i}\right), & \text { if } \beta<\alpha \\
\max \left(c_{i}\right), & \text { if } \alpha<\beta, \\
\max \left(c_{i}\right)-\max \left(c_{z i}\right), & \text { if } \alpha=\beta
\end{array}\right.
\end{aligned}
$$

which is non-zero and finite in all cases (recall $c_{z i}<0, c_{i}>0$ for all $i$ ). Also $\left\|R_{n}\right\|^{-t}\left\|R_{n z}\right\|^{t}=o(1)$ and $\left\|R_{n z}\right\|^{t-k_{n}}\left\|R_{n}\right\|^{k_{n}-t}=o(1)$ where the second equality holds because

$$
\left\|R_{n z}\right\|^{t-k_{n}}\left\|R_{n}\right\|^{k_{n}-t}=O\left(e^{\max \left(c_{z i}\right) \frac{t-k_{n}}{n^{\beta}}} e^{-\max \left(c_{i}\right) \frac{t-k_{n}}{n^{\alpha}}}\right),
$$

and $\frac{k_{n}^{\prime}}{n^{\alpha \wedge \beta}} \leq \frac{t-k_{n}}{n^{\alpha \wedge \beta}} \rightarrow \infty$ by the given rate condition. Hence $n^{-\left(\frac{\alpha}{2}+\alpha \wedge \beta\right)} R_{n}^{-t} \sum_{j=1}^{k_{n}} R_{n z}^{t-j} x_{j-1}=o_{p}(1)$.

Now we have

$$
\begin{aligned}
\frac{1}{n^{\frac{\alpha}{2}+\alpha \wedge \beta}} R_{n}^{-t} \psi_{n t} & =\frac{1}{n^{\frac{\alpha}{2}+\alpha \wedge \beta}} R_{n}^{-t} \sum_{j=k_{n}+1}^{t} R_{n z}^{t-j} x_{j-1}+o_{p}(1) \\
& =\frac{1}{n^{\alpha \wedge \beta}} R_{n}^{-t} \sum_{j=k_{n}+1}^{t} R_{n z}^{t-j} R_{n}^{(j-1)}\left(R_{n}^{-(j-1)} \frac{x_{j-1}}{n^{\alpha / 2}}\right)+o_{p}(1) \\
& =\left(\frac{1}{n^{\alpha \wedge \beta}} R_{n}^{-t} \sum_{j=k_{n}+1}^{t} R_{n z}^{t-j} R_{n}^{(j-1)}\right)\left(\tilde{Y}_{C}+o_{p}(1)\right) \text { (using lemma 2.2) } \\
& =\left(\frac{1}{n^{\alpha \wedge \beta}} R_{n}^{-t} \sum_{j=1}^{t} R_{n z}^{t-j} R_{n}^{(j-1)}\right)\left(\tilde{Y}_{C}+o_{p}(1)\right), \text { (putting back the negligible front sum) }
\end{aligned}
$$


Note that

$$
\begin{aligned}
& \frac{1}{n^{(\alpha \wedge \beta)}} \sum_{j=1}^{t} R_{n z}^{t-j} R_{n}^{j-1-t} \\
= & \frac{1}{n^{(\alpha \wedge \beta)}} \operatorname{diag}\left(\sum_{j=1}^{t}\left(1+\frac{c_{z 1}}{n^{\beta}}\right)^{t-j}\left(1+\frac{c_{1}}{n^{\alpha}}\right)^{j-1-t}, \ldots, \sum_{j=1}^{n}\left(1+\frac{c_{z K}}{n^{\beta}}\right)^{t-j}\left(1+\frac{c_{K}}{n^{\alpha}}\right)^{j-1-t}\right) .
\end{aligned}
$$

For all $i$

$$
\begin{aligned}
& \frac{1}{n^{(\alpha \wedge \beta)}} \sum_{j=1}^{t}\left(1+\frac{c_{z i}}{n^{\beta}}\right)^{t-j}\left(1+\frac{c_{i}}{n^{\alpha}}\right)^{j-1-t}=\frac{1}{n^{(\alpha \wedge \beta)}}\left(1+\frac{c_{z i}}{n^{\beta}}\right)^{t-1}\left(1+\frac{c_{i}}{n^{\alpha}}\right)^{-t} \frac{\left\{\left(1+\frac{c_{z i}}{n^{\beta}}\right)^{-t}\left(1+\frac{c_{i}}{n^{\alpha}}\right)^{t}-1\right\}}{\left(1+\frac{c_{z i}}{n^{\beta}}\right)^{-1}\left(1+\frac{c_{i}}{n^{\alpha}}\right)-1} \\
= & \frac{1}{n^{(\alpha \wedge \beta)}} \frac{\left\{\left(1+\frac{c_{z i}}{n^{\beta}}\right)^{-1}-\left(1+\frac{c_{z i}}{n^{\beta}}\right)^{t-1}\left(1+\frac{c_{i}}{n^{\alpha}}\right)^{-t}\right\}}{\left(1+\frac{c_{z i}}{n^{\beta}}\right)^{-1}\left(1+\frac{c_{i}}{n^{\alpha}}\right)-1}=\frac{1+o(1)}{n^{(\alpha \wedge \beta)}\left(\frac{c_{i}}{n^{\alpha}}-\frac{c_{z i}}{n^{\beta}}-\frac{c c_{z i}}{n^{\alpha+\beta}}\right)} \\
\rightarrow & -\frac{1}{c_{z i}} \text { if } \beta<\alpha, \frac{1}{c_{i}} \text { if } \alpha<\beta \text { and } \frac{1}{c_{i}-c_{z i}} \text { if } \alpha=\beta .
\end{aligned}
$$

Hence,

$$
\frac{1}{n^{(\alpha \wedge \beta)}} R_{n}^{-t} \sum_{j=1}^{t} R_{n z}^{t-j} R_{n}^{(j-1)} \rightarrow\left\{\begin{array}{cc}
-C_{z}^{-1}, & \text { if } \beta<\alpha \\
C^{-1}, & \text { if } \alpha<\beta \\
\left(C-C_{z}\right)^{-1}, & \text { if } \alpha=\beta
\end{array}\right\}=: C_{z \alpha \beta},
$$

giving the required result.

Proof of Lemma 2.4. Part (1). From 2.10, we have

$$
\begin{aligned}
\frac{1}{n^{(\alpha \wedge \beta)}} \sum_{t=1}^{n} u_{0 t} \tilde{z}_{t}^{\prime} R_{n}^{-n} & =\frac{1}{n^{(\alpha \wedge \beta)}} \sum_{t=1}^{n} u_{0 t} z_{t}^{\prime} R_{n}^{-n}+\frac{1}{n^{\alpha+(\alpha \wedge \beta)}} \sum_{t=1}^{n} u_{0 t} \psi_{n t}^{\prime} R_{n}^{-n} C \\
& =\frac{1}{n^{\alpha+(\alpha \wedge \beta)}} \sum_{t=1}^{n} u_{0 t} \psi_{n t}^{\prime} R_{n}^{-n} C+o_{p}(1)
\end{aligned}
$$

where the last equality holds because $R_{n}^{-n}$ dominates the order of magnitude of $\sum_{t=1}^{n} u_{0 t} z_{t}^{\prime}=O_{p}(n)$ (c.f., Magdalinos and Phillips, 2009a, equation (10)).

To use lemma 2.3 we first show that $n^{-(\alpha+(\alpha \wedge \beta))} \sum_{t=1}^{k_{n}+k_{n}^{\prime}-1} u_{0 t} \psi_{n t}^{\prime} R_{n}^{-n}=o_{p}(1)$ for $k_{n}$ and $k_{n}^{\prime}$ satisfying the conditions of lemma 2.3. Note that

$$
\begin{aligned}
& \left\|\frac{1}{n^{\alpha+(\alpha \wedge \beta)}} \sum_{t=1}^{k_{n}+k_{n}^{\prime}-1} u_{0 t} \psi_{n t}^{\prime} R_{n}^{-n}\right\|_{L_{1}}=\left\|\frac{1}{n^{\frac{\alpha}{2}}} \sum_{t=1}^{k_{n}+k_{n}^{\prime}-1} u_{0 t}\left(\frac{1}{n^{\frac{\alpha}{2}+(\alpha \wedge \beta)}} R_{n}^{-t} \psi_{n t}\right)^{\prime} R_{n}^{t-n}\right\|_{L_{1}} \\
\leq & \left(\sup _{t}\left\|u_{0 t}\right\|_{L_{2}}\right)\left(\sup _{t}\left\|\frac{1}{n^{\frac{\alpha}{2}+(\alpha \wedge \beta)}} R_{n}^{-t} \psi_{n t}\right\|_{L_{2}}\right) \frac{\left\|R_{n}\right\|^{-n}}{n^{\frac{\alpha}{2}}} \sum_{t=1}^{k_{n}+k_{n}^{\prime}-1}\left\|R_{n}\right\|^{t} \text { and using (2.15), }
\end{aligned}
$$




$$
=O\left(\frac{n^{\frac{\alpha}{2}}\left\|R_{n}\right\|^{k_{n}+k_{n}^{\prime}-n}}{n^{\alpha}\left(1-\left\|R_{n}\right\|\right)}\right)=O\left(n^{\frac{\alpha}{2}}\left\|R_{n}\right\|^{k_{n}+k_{n}^{\prime}-n}\right)=o(1),
$$

because of the exponentially fast convergence of $\left\|R_{n}\right\|^{k_{n}+k_{n}^{\prime}-n} \rightarrow 0$.

Hence, we have $n^{-(\alpha+\alpha \wedge \beta)} \sum_{t=1}^{k_{n}+k_{n}^{\prime}-1} u_{0 t} \psi_{n t}^{\prime} R_{n}^{-n}=o_{p}(1)$.

Using the same sum splitting argument as in lemma 2.3,

$$
\begin{aligned}
& \operatorname{vec}\left(\frac{1}{n^{\alpha+(\alpha \wedge \beta)}} \sum_{t=1}^{n} u_{0 t} \psi_{n t}^{\prime} R_{n}^{-n}\right)=v e c\left(\frac{1}{n^{\alpha+(\alpha \wedge \beta)}} \sum_{t=k_{n}+k_{n}^{\prime}}^{n} u_{0 t} \psi_{n t}^{\prime} R_{n}^{-n}\right)+o_{p}(1) \\
= & \operatorname{vec}\left(\frac{1}{n^{\frac{\alpha}{2}}} \sum_{t=k_{n}+k_{n}^{\prime}}^{n} u_{0 t}\left(\frac{1}{n^{\frac{\alpha}{2}+(\alpha \wedge \beta)}} R_{n}^{-t} \psi_{n t}\right)^{\prime}\left(R_{n}^{t-n}\right)^{\prime}\right)+o_{p}(1) \\
= & \operatorname{vec}\left(\frac{1}{n^{\frac{\alpha}{2}}} \sum_{t=k_{n}+k_{n}^{\prime}}^{n} u_{0 t}\left(C_{z \alpha \beta} \tilde{Y}_{C}+o_{p}(1)\right)^{\prime}\left(R_{n}^{t-n}\right)^{\prime}\right)+o_{p}(1) \quad \text { (using lemma 2.3) } \\
= & \operatorname{vec}\left(\frac{1}{n^{\frac{\alpha}{2}}} \sum_{t=k_{n}+k_{n}^{\prime}}^{n} u_{0 t}\left(\tilde{Y}_{C}\right)^{\prime}\left(R_{n}^{t-n} C_{z \alpha \beta}\right)^{\prime}\right)+o_{p}(1) \\
= & \left(\frac{1}{n^{\frac{\alpha}{2}}} \sum_{t=k_{n}+k_{n}^{\prime}}^{n} R_{n}^{-(n-t)} C_{z \alpha \beta} \otimes u_{0 t}\right)\left(\tilde{Y}_{C}\right)+o_{p}(1) \\
= & \left(C_{z \alpha \beta} \otimes I_{m}\right)\left(\frac{1}{n^{\frac{\alpha}{2}}} \sum_{t=1}^{n} R_{n}^{-(n-t)} \otimes u_{0 t}\right)\left(\tilde{Y}_{C}\right)+o_{p}(1) \quad \text { (putting back the front sum) } \\
\Longrightarrow & \left(C_{z \alpha \beta} \otimes I_{m}\right) \times M N\left(0, \int_{0}^{\infty} e^{-p C} \tilde{Y}_{C} \tilde{Y}_{C}^{\prime} e^{-p C} d p \otimes \Omega_{00}\right)
\end{aligned}
$$

where the last step comes from the same procedure as in equations (22)-(26) of Magdalinos and Phillips (2009a). Finally,

$$
\begin{aligned}
\operatorname{vec}\left(\frac{1}{n^{(\alpha \wedge \beta)}} \sum_{t=1}^{n} u_{0 t} \tilde{z}_{t}^{\prime} R_{n}^{-n}\right) & =\left(C \otimes I_{m}\right) \operatorname{vec}\left(\frac{1}{n^{\alpha+(\alpha \wedge \beta)}} \sum_{t=1}^{n} u_{0 t} \psi_{n t}^{\prime} R_{n}^{-n}\right)+o_{p}(1) \\
& \Longrightarrow\left(C C_{z \alpha \beta} \otimes I_{m}\right) \times M N\left(0, \int_{0}^{\infty} e^{-p C} Y_{C} Y_{C}^{\prime} e^{-p C} d p \otimes \Omega_{00}\right)
\end{aligned}
$$

where we have used $Y_{C}$ instead of its distributional copy $\tilde{Y}_{C}$ since we are concerned with weak convergence in the original probability space from this point onwards. 
Part (2). From $\sum x_{t} \tilde{z}_{t}^{\prime}=\sum x_{t} z_{t}^{\prime}+n^{-\alpha} \sum_{t=1}^{n} x_{t} \psi_{n t}^{\prime} C$, we have

$$
\begin{aligned}
\frac{1}{n^{\alpha+(\alpha \wedge \beta)} \sum R_{n}^{-n} x_{t} \tilde{z}_{t}^{\prime} R_{n}^{-n}} & =\frac{1}{n^{\alpha+(\alpha \wedge \beta)}} \sum R_{n}^{-n} x_{t} z_{t}^{\prime} R_{n}^{-n}+\frac{1}{n^{2 \alpha+(\alpha \wedge \beta)}} \sum_{t=1}^{n} R_{n}^{-n} x_{t} \psi_{n t}^{\prime} R_{n}^{-n} C \\
& =\frac{1}{n^{2 \alpha+(\alpha \wedge \beta)}} \sum_{t=1}^{n} R_{n}^{-n} x_{t} \psi_{n t}^{\prime} R_{n}^{-n} C+o_{p}(1)
\end{aligned}
$$

as in Part (1) and using the same sum splitting argument again with lemma 2.2 and 2.3,

$$
\begin{aligned}
\frac{1}{n^{2 \alpha+(\alpha \wedge \beta)} \sum_{t=1}^{n} R_{n}^{-n} x_{t} \psi_{n t}^{\prime} R_{n}^{-n}}=\frac{1}{n^{2 \alpha+(\alpha \wedge \beta)}} \sum_{t=k_{n}+k_{n}^{\prime}}^{n} R_{n}^{-n} x_{t} \psi_{n t}^{\prime} R_{n}^{-n}+o_{p}(1) \\
=\frac{1}{n^{\alpha}} \sum_{t=k_{n}+k_{n}^{\prime}}^{n} R_{n}^{-(n-t)}\left(\frac{R_{n}^{-t}}{n^{\frac{\alpha}{2}}} x_{t}\right)\left(\frac{R_{n}^{-t}}{n^{\frac{\alpha}{2}+(\alpha \wedge \beta)}} \psi_{n t}\right)^{\prime} R_{n}^{-(n-t)}+o_{p}(1) \\
=\frac{1}{n^{\alpha}} \sum_{t=k_{n}+k_{n}^{\prime}}^{n} R_{n}^{-(n-t)}\left(\tilde{Y}_{C}\right)\left(\tilde{Y}_{C}\right)^{\prime} R_{n}^{-(n-t)} C_{z \alpha \beta}+o_{p}(1) \\
=\frac{1}{n^{\alpha}} \sum_{t=1}^{n} R_{n}^{-(n-t)}\left(\tilde{Y}_{C}\right)\left(\tilde{Y}_{C}\right)^{\prime} R_{n}^{-(n-t)} C_{z \alpha \beta}+o_{p}(1) \\
\Longrightarrow \int_{0}^{\infty} e^{-p C} Y_{C} Y_{C}^{\prime} e^{-p C} d p C_{z \alpha \beta} .
\end{aligned}
$$

It follows that

$$
\frac{1}{n^{\alpha+(\alpha \wedge \beta)}} \sum R_{n}^{-n} x_{t} \tilde{z}_{t}^{\prime} R_{n}^{-n} \Longrightarrow \int_{0}^{\infty} e^{-p C} Y_{C} Y_{C}^{\prime} e^{-p C} d p C C_{z \alpha \beta}
$$

Proof of Theorem 2.2. We have the following limit theory for the IVX estimator $\hat{A}_{n}=$ $\left(Y^{\prime} \tilde{Z}\right)\left(X^{\prime} \tilde{Z}\right)^{-1}$ without the bias correction shown in 2.12 . Joint convergence of the numerator and denominator in lemma 2.4 parts (1) and (2) is established as in Magdalinos and Phillips (2009a, Proof of Theorem 4.1). Using these results with $\hat{A}_{n}-A=\left(U_{0}^{\prime} \tilde{Z}\right)\left(X^{\prime} \tilde{Z}\right)^{-1}$ we have

$$
\begin{aligned}
\operatorname{vec}\left[n^{\alpha}\left(\hat{A}_{n}-A\right) R_{n}^{n}\right]= & {\left[\left\{\left(\frac{1}{n^{\alpha+(\alpha \wedge \beta)}} \sum R_{n}^{-n} x_{t} \tilde{z}_{t}^{\prime} R_{n}^{-n}\right)^{\prime}\right\}^{-1} \otimes I_{m}\right] \operatorname{vec}\left(\frac{1}{n^{(\alpha \wedge \beta)}} \sum_{t=1}^{n} u_{0 t} \tilde{z}_{t}^{\prime} R_{n}^{-n}\right) } \\
\Longrightarrow & {\left[\left(\left\{\tilde{\Psi}_{y y} \times\left(C C_{z \alpha \beta}\right)\right\}^{\prime}\right)^{-1} \otimes I_{m}\right] } \\
& \times\left(C C_{z \alpha \beta} \otimes I_{m}\right) \times M N\left(0, \tilde{\Psi}_{y y} \otimes \Omega_{00}\right) \\
\equiv & M N\left(0,\left(\tilde{\Psi}_{y y}\right)^{-1} \otimes \Omega_{00}\right) .
\end{aligned}
$$


The bias corrected IVX estimator given in 2.12 is asymptotically equivalent to the uncorrected estimator $\hat{A}_{n}$ due to the signal strength of the $X^{\prime} \tilde{Z}$ matrix, i.e.,

$$
\hat{A}_{n}-\tilde{A}_{n}=n \widehat{\Delta}_{0 x}\left(X^{\prime} \tilde{Z}\right)^{-1}=O_{p}\left(\frac{n}{n^{\alpha+(\alpha \wedge \beta)}\left\|R_{n}\right\|^{2 n}}\right)=o_{p}(1),
$$

and

$$
\begin{aligned}
n^{\alpha}\left(\tilde{A}_{n}-A\right) R_{n}^{n} & =n^{\alpha}\left(\hat{A}_{n}-A\right) R_{n}^{n}+O_{p}\left(\frac{n^{1-(\alpha \wedge \beta)}}{\left\|R_{n}\right\|^{n}}\right) \\
& =n^{\alpha}\left(\hat{A}_{n}-A\right) R_{n}^{n}+o_{p}(1) .
\end{aligned}
$$

As a result, the same limit theory holds regardless of the bias correction, proving theorem 2.2.

The following lemma helps in characterizing the variance matrix asymptotics for (2.11).

Lemma $6.2 \frac{1}{n^{2(\alpha \wedge \beta)}} \sum R_{n}^{-n} \tilde{z}_{t} \tilde{z}_{t}^{\prime} R_{n}^{-n} \Longrightarrow C C_{z \alpha \beta} \tilde{\Psi}_{y y} C C_{z \alpha \beta}$.

Proof. We have the decomposition of the sample moment matrix

$$
\begin{aligned}
\sum \tilde{z}_{t} \tilde{z}_{t}^{\prime} & =\sum\left(z_{t}+\frac{C}{n^{\alpha}} \psi_{n t}\right)\left(z_{t}+\frac{C}{n^{\alpha}} \psi_{n t}\right)^{\prime} \\
& =\sum z_{t} z_{t}^{\prime}+\frac{C}{n^{\alpha}} \sum \psi_{n t} z_{t}^{\prime}+\frac{1}{n^{\alpha}} \sum z_{t} \psi_{n t}^{\prime} C+\frac{1}{n^{2 \alpha}} \sum C \psi_{n t} \psi_{n t}^{\prime} C^{\prime} .
\end{aligned}
$$

By the same methods used in lemma 2.3 and 2.4, it is straightforward to show that

$$
\begin{aligned}
& \frac{1}{n^{2 \alpha+2(\alpha \wedge \beta)}} \sum_{t=1}^{n} R_{n}^{-n} \psi_{n t} \psi_{n t}^{\prime} R_{n}^{-n} \\
= & \frac{1}{n^{\alpha}} \sum_{t=1}^{n} R_{n}^{-(n-t)}\left(\frac{1}{n^{\frac{\alpha}{2}+(\alpha \wedge \beta)}} R_{n}^{-t} \psi_{n t}\right)\left(\frac{1}{n^{\frac{\alpha}{2}+(\alpha \wedge \beta)}} R_{n}^{-t} \psi_{n t}\right)^{\prime} R_{n}^{-(n-t)} \\
= & \frac{1}{n^{\alpha}} \sum_{t=k_{n}+k_{n}^{\prime}}^{n} R_{n}^{-(n-t)}\left(\frac{1}{n^{\frac{\alpha}{2}+(\alpha \wedge \beta)}} R_{n}^{-t} \psi_{n t}\right)\left(\frac{1}{n^{\frac{\alpha}{2}+(\alpha \wedge \beta)}} R_{n}^{-t} \psi_{n t}\right)^{\prime} R_{n}^{-(n-t)}+o_{p}(1) \\
= & \frac{1}{n^{\alpha}} \sum_{t=k_{n}+k_{n}^{\prime}}^{n} R_{n}^{-(n-t)}\left(C_{z \alpha \beta} \tilde{Y}_{C}\right)\left(C_{z \alpha \beta} \tilde{Y}_{C}\right)^{\prime} R_{n}^{-(n-t)}+o_{p}(1)
\end{aligned}
$$




$$
\begin{aligned}
& =\left(C_{z \alpha \beta}\right)\left(\frac{1}{n^{\alpha}} \sum_{t=k_{n}+k_{n}^{\prime}}^{n} R_{n}^{-(n-t)}\left(\tilde{Y}_{C}\right)\left(\tilde{Y}_{C}\right)^{\prime} R_{n}^{-(n-t)}\right)\left(C_{z \alpha \beta}\right)+o_{p}(1) \\
& =\left(C_{z \alpha \beta}\right)\left(\frac{1}{n^{\alpha}} \sum_{t=1}^{n} R_{n}^{-(n-t)}\left(\tilde{Y}_{C}\right)\left(\tilde{Y}_{C}\right)^{\prime} R_{n}^{-(n-t)}\right)\left(C_{z \alpha \beta}\right)+o_{p}(1) \\
& \Longrightarrow\left(C_{z \alpha \beta}\right) \int_{0}^{\infty} e^{-p C} Y_{C} Y_{C}^{\prime} e^{-p C} d p\left(C_{z \alpha \beta}\right) .
\end{aligned}
$$

This term clearly dominates all the other terms since $z_{t}$ is mildly integrated. Hence

$$
\begin{aligned}
\frac{1}{n^{2(\alpha \wedge \beta)} \sum R_{n}^{-n} \tilde{z}_{t} \tilde{z}_{t}^{\prime} R_{n}^{-n}} & =C\left(\frac{1}{n^{2 \alpha+2(\alpha \wedge \beta)}} \sum_{t=1}^{n} R_{n}^{-n} \psi_{n t} \psi_{n t}^{\prime} R_{n}^{-n}\right) C+o_{p}(1) \\
& \Longrightarrow\left(C C_{z \alpha \beta}\right) \int_{0}^{\infty} e^{-p C} Y_{C} Y_{C}^{\prime} e^{-p C} d p\left(C C_{z \alpha \beta}\right) \\
& \equiv C C_{z \alpha \beta} \tilde{\Psi}_{y y} C C_{z \alpha \beta} .
\end{aligned}
$$

The robust chi-square limit theory of the self-normalized IVX estimator follows and is given in the next proof.

Proof of Theorem 2.3. We have the following limit theory for the variance estimator,

$$
\begin{aligned}
& n^{2 \alpha}\left(R_{n}^{n} \otimes I_{m}\right)\left[\left(X^{\prime} P_{\widetilde{Z}} X\right)^{-1} \otimes \hat{\Omega}_{00}\right]\left(R_{n}^{n} \otimes I_{m}\right) \\
& =\left[\frac{1}{n^{2 \alpha}} R_{n}^{-n}\left\{X^{\prime} \tilde{Z}\left(\tilde{Z}^{\prime} \tilde{Z}\right)^{-1} \tilde{Z}^{\prime} X\right\} R_{n}^{-n}\right]^{-1} \otimes \hat{\Omega}_{00}
\end{aligned}
$$

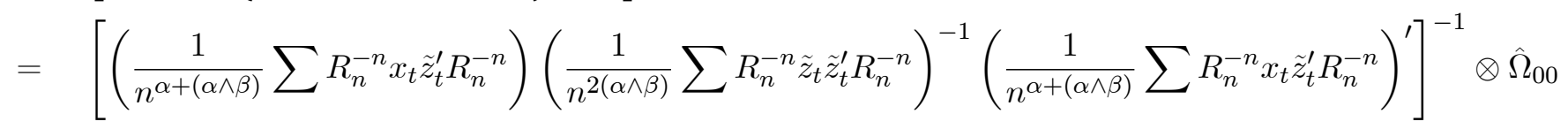

$$
\begin{aligned}
& \Longrightarrow\left[\left(\tilde{\Psi}_{y y}\left(C C_{z \alpha \beta}\right)\right)\left(\left(C C_{z \alpha \beta}\right) \tilde{\Psi}_{y y}\left(C C_{z \alpha \beta}\right)\right)^{-1}\left(\tilde{\Psi}_{y y}\left(C C_{z \alpha \beta}\right)\right)^{\prime}\right]^{-1} \otimes \Omega_{00} \\
& \equiv \tilde{\Psi}_{y y}^{-1} \otimes \Omega_{00},
\end{aligned}
$$

and again the weak convergence is joint with that of the estimator components. Hence

$$
\begin{aligned}
& \operatorname{vec}\left(\tilde{A}_{n}-A\right)^{\prime}\left[\left(X^{\prime} P_{\widetilde{Z}} X\right)^{-1} \otimes \hat{\Omega}_{00}\right]^{-1} \operatorname{vec}\left(\tilde{A}_{n}-A\right) \\
= & \operatorname{vec}\left(\tilde{A}_{n}-A\right)^{\prime}\left(R_{n}^{n} \otimes I_{m}\right)\left(R_{n}^{n} \otimes I_{m}\right)^{-1}\left[\left(X^{\prime} P_{\widetilde{Z}} X\right)^{-1} \otimes \hat{\Omega}_{00}\right]^{-1}\left(R_{n}^{n} \otimes I_{m}\right)^{-1}\left(R_{n}^{n} \otimes I_{m}\right) \operatorname{vec}\left(\tilde{A}_{n}-A\right) \\
= & \left\{\operatorname{vec}\left[n^{\alpha}\left(\tilde{A}_{n}-A\right) R_{n}^{n}\right]\right\}^{\prime}\left[n^{2 \alpha}\left(R_{n}^{n} \otimes I_{m}\right)\left[\left(X^{\prime} P_{\widetilde{Z}} X\right)^{-1} \otimes \hat{\Omega}_{00}\right]\left(R_{n}^{n} \otimes I_{m}\right)\right]^{-1} \operatorname{vec}\left[n^{\alpha}\left(\tilde{A}_{n}-A\right) R_{n}^{n}\right] \\
\Longrightarrow & \chi^{2}(m K),
\end{aligned}
$$


proving theorem 2.3 .

Proof of Proposition 2.4. Note that

$$
\frac{1}{n^{\alpha+(\alpha \wedge \beta)}} \sum R_{n}^{-n} x_{t}^{\mu} \tilde{z}_{t}^{\prime} R_{n}^{-n}=\frac{1}{n^{\alpha+(\alpha \wedge \beta)}} \sum R_{n}^{-n} x_{t} \tilde{z}_{t}^{\prime} R_{n}^{-n}-\frac{1}{n^{1+\alpha+(\alpha \wedge \beta)}} \sum R_{n}^{-n} x_{t} \sum \tilde{z}_{t}^{\prime} R_{n}^{-n} .
$$

Using the fact that the IVX remainder is the dominating order in the (I5) case, we have

$$
\begin{aligned}
& \frac{1}{n^{1+\alpha+(\alpha \wedge \beta)}} \sum R_{n}^{-n} x_{t} \sum \tilde{z}_{t}^{\prime} R_{n}^{-n}=\frac{1}{n^{1+\alpha+(\alpha \wedge \beta)}} \sum R_{n}^{-n} x_{t} \sum z_{t}^{\prime} R_{n}^{-n}+\frac{1}{n^{1+2 \alpha+(\alpha \wedge \beta)}} \sum R_{n}^{-n} x_{t} \sum \psi_{n t}^{\prime} R_{n}^{-n} C \\
& =\frac{1}{n^{1+2 \alpha+(\alpha \wedge \beta)}} \sum R_{n}^{-n} x_{t} \sum \psi_{n t}^{\prime} R_{n}^{-n} C+o_{p}(1) \text {, }
\end{aligned}
$$

and using Lemma 2.2 and 2.3, we deduce that

$$
\begin{aligned}
\frac{1}{n^{1+2 \alpha+(\alpha \wedge \beta)}} \sum R_{n}^{-n} x_{t} \sum \psi_{n t}^{\prime} R_{n}^{-n} C & = \\
\frac{n^{3 \alpha+(\alpha \wedge \beta)}}{n^{1+2 \alpha+(\alpha \wedge \beta)}}\left\{n^{-\alpha} \sum R_{n}^{-n}\left(\frac{x_{t}}{n^{\alpha / 2}} R_{n}^{-t}\right) R_{n}^{t}\right\}\left\{n^{-\alpha} \sum\left(\frac{\psi_{n t}}{n^{\alpha / 2+(\alpha \wedge \beta)}} R_{n}^{-t}\right)^{\prime} R_{n}^{-n} C\right\} & =O_{p}\left(\frac{1}{n^{1-\alpha}}\right)=o_{p}(1),
\end{aligned}
$$

which gives the required result.

\subsection{The Case of Mixed Roots: (I6)}

We provided the proofs for Theorem 3.1 and 3.2, while the proofs for Lemma 3.1, 3.2 and 6.3 are available from Phillips and Lee (2015c).

Proof of Theorem 3.1. We write the scaled estimation error as

$$
C_{n}(\tilde{a}-a)=\left(D_{n}^{-1}\left[\begin{array}{ccc}
\sum_{t=1}^{n} \tilde{z}_{1 t} x_{1 t} & \sum_{t=1}^{n} \tilde{z}_{1 t} x_{2 t} \\
\sum_{t=1}^{n} \tilde{z}_{2 t} x_{1 t} & \sum_{t=1}^{n} \tilde{z}_{2 t} x_{2 t}
\end{array}\right] C_{n}^{-1}\right)^{-1} D_{n}^{-1}\left[\begin{array}{c}
\sum_{t=1}^{n} \tilde{z}_{1 t} u_{0 t}-n \hat{\Delta}_{0 x 1} \\
\sum_{t=1}^{n} \tilde{z}_{2 t} u_{0 t}-n \hat{\Delta}_{0 x 2}
\end{array}\right]
$$

and using Lemma 3.2,

$$
\left[\begin{array}{c}
n^{\frac{1+\left(\alpha_{1} \wedge \beta\right)}{2}}\left(\tilde{a}_{1}-a_{1}\right) \\
\theta_{n}^{n} n^{\alpha_{2}}\left(\tilde{a}_{2}-a_{2}\right)
\end{array}\right]=\left[\begin{array}{c}
\left(\frac{1}{n^{1+\left(\alpha_{1} \wedge \beta\right)}} \sum_{t=1}^{n} \tilde{z}_{1 t} x_{1 t}\right)^{-1} \frac{1}{n^{\frac{1+\left(\alpha_{1} \wedge \beta\right)}{2}}} \sum_{t=1}^{n}\left(\tilde{z}_{1 t} u_{0 t}-\hat{\Delta}_{0 x 1}\right) \\
\left(\frac{1}{\theta_{n}^{2 n} n^{\alpha_{2}+\left(\alpha_{2} \wedge \beta\right)}} \sum_{t=1}^{n} \tilde{z}_{2 t} x_{2 t}\right)^{-1} \frac{1}{\theta_{n}^{n} n^{\left(\alpha_{2} \wedge \beta\right)}} \sum_{t=1}^{n}\left(\tilde{z}_{2 t} u_{0 t}-\hat{\Delta}_{0 x 2}\right)
\end{array}\right]+o_{p}(1)
$$

The limit theory of $n^{\frac{1+\left(\alpha_{1} \wedge \beta\right)}{2}}\left(\tilde{a}_{1}-a_{1}\right)$ is exactly a scalar version of Theorem 3.4 and 3.7 from MP 
so that

$$
\Longrightarrow\left\{\begin{array}{cr}
n^{\frac{1+\left(\alpha_{1} \wedge \beta\right)}{2}\left(\tilde{a}_{1}-a_{1}\right)} \\
\left.M N\left(0,\left\{\frac{1}{-c_{z 1}}\left(\Omega_{11}+\int_{0}^{1} B_{1} d B_{1}\right)\right\}^{-2}\left(\frac{1}{-2 c_{z 1}} \Omega_{11}\right) \Omega_{00}\right)\right) & \text { if } x_{1 t} \text { is unit root - I(1) } \\
\left.M N\left(0,\left\{\frac{1}{-c_{z 1}}\left\{\Omega_{11}+\int_{0}^{1} J_{x}^{c 1} d J_{x}^{c 1}\right\}\right\}^{-2}\left(\frac{1}{-2 c_{z 1}} \Omega_{11}\right) \Omega_{00}\right)\right) & \text { if } x_{1 t} \text { is local to unity - (I2) or (I4) } \\
N\left(0,\left(\frac{1}{-2 c_{z 1}} \Omega_{11}\right)^{-1} \Omega_{00}\right) & \text { if } x_{1 t} \text { is mildly integrated - (I3) with } \beta<\alpha_{1} \\
N\left(0, \frac{\left(c_{1}+c_{z 1}\right)^{2}}{c_{1}^{2}}\left(\frac{1}{-2 c_{1}} \Omega_{11}\right)^{-1} \Omega_{00}\right) & \text { if } x_{1 t} \text { is mildly integrated - (I3) with } \beta=\alpha_{1} \\
N\left(0,\left(\frac{1}{-2 c_{1}} \Omega_{11}\right)^{-1} \Omega_{00}\right) & \text { if } x_{1 t} \text { is mildly integrated - (I3) with } \alpha_{1}<\beta
\end{array}\right.
$$

By defining

$$
\tilde{\Phi}_{11}^{-1}=\left\{\begin{array}{cc}
\left\{\frac{1}{-c_{z 1}}\left(\Omega_{11}+\int_{0}^{1} B_{1} d B_{1}\right)\right\}^{-2}\left(\frac{1}{-2 c_{z 1}} \Omega_{11}\right) & \text { if } x_{1 t} \text { is unit root - I(1) } \\
\left\{\frac{1}{-c_{z 1}}\left\{\Omega_{11}+\int_{0}^{1} J_{x}^{c 1} d J_{x}^{c 1}\right\}\right\}^{-2}\left(\frac{1}{-2 c_{z 1}} \Omega_{11}\right) & \text { if } x_{1 t} \text { is local to unity - (I2) or (I4) } \\
\left(\frac{1}{-2 c_{z}} \Omega_{11}\right)^{-1} & \text { if } x_{1 t} \text { is mildly integrated - (I3) with } \beta<\alpha_{1} \\
\frac{\left(c_{1}+c_{z 1}\right)^{2}}{c_{1}^{2}}\left(\frac{1}{-2 c_{1}} \Omega_{11}\right)^{-1} & \text { if } x_{1 t} \text { is mildly integrated - (I3) with } \beta=\alpha_{1} \\
\left(\frac{1}{-2 c_{1}} \Omega_{11}\right)^{-1} & \text { if } x_{1 t} \text { is mildly integrated - (I3) with } \alpha_{1}<\beta
\end{array}\right.
$$

we have

$$
\left.n^{\frac{1+\left(\alpha_{1} \wedge \beta\right)}{2}}\left(\tilde{a}_{1}-a_{1}\right) \Longrightarrow M N\left(0, \tilde{\Phi}_{11}^{-1} \Omega_{00}\right)\right)
$$

From lemma 2.4-(1), we have

$$
\frac{1}{\theta_{n}^{n} n^{\left(\alpha_{2} \wedge \beta\right)}} \sum_{t=1}^{n} \tilde{z}_{2 t} u_{0 t} \Longrightarrow c_{z_{2} \alpha \beta} c_{2} M N\left(0, \tilde{\Psi}_{22} \Omega_{00}\right)
$$

and together with lemma 3.2 this leads to

$$
\theta_{n}^{n} n^{\alpha_{2}}\left(\tilde{a}_{2}-a_{2}\right) \Longrightarrow M N\left(0, \tilde{\Psi}_{22}^{-1} \Omega_{00}\right)
$$

which is a special case of theorem 2.2. Joint convergence and asymptotic independence follow from lemma 3.1, thereby completing the proof.

The same mechanism for variance estimation, as shown in the following lemma, now leads to nuisance parameter free inference in the corresponding self-normalized test statistics.

Lemma 6.3 $\left[C_{n}\left(X^{\prime} P_{\widetilde{Z}} X\right)^{-1} C_{n} \hat{\Omega}_{00}\right]^{-1} \Longrightarrow\left[\left[\begin{array}{cc}\tilde{\Phi}_{11} \Omega_{00}^{-1} & 0 \\ 0 & \tilde{\Psi}_{22} \Omega_{00}^{-1}\end{array}\right]\right]$.

Proof of Theorem 3.2. Using Lemma 3.2, Theorem 3.1 and lemma 6.3, we have directly by 
continuous mapping

$$
\begin{aligned}
& \left(\tilde{a}-a_{0}\right)^{\prime}\left[\left(X^{\prime} P_{\widetilde{Z}} X\right)^{-1} \hat{\Omega}_{00}\right]^{-1}\left(\tilde{a}-a_{0}\right) \\
= & \left(\tilde{a}-a_{0}\right)^{\prime} C_{n}\left[C_{n}\left(X^{\prime} P_{\widetilde{Z}} X\right)^{-1} C_{n} \hat{\Omega}_{00}\right]^{-1} C_{n}(\tilde{a}-a) \Longrightarrow \chi^{2}(2) .
\end{aligned}
$$

Acknowledgement We thank four referees and the Guest Editors for helpful comments and guidance on revising the original version of this paper. Phillips acknowledges support under NSF Grant No. SES 12-58258.

\section{$7 \quad$ References}

Andrews, D. W., \& Guggenberger, P. (2014). A Conditional-Heteroskedasticity-Robust Confidence Interval for the Autoregressive Parameter. Review of Economics and Statistics, 96(2), 376-381.

Campbell, J. and M. Yogo (2006). "Efficient tests of stock return predictability," Journal of Financial Economics, 81(1), 27-60.

Diebold, Francis X., and Atsushi Inoue. "Long memory and regime switching." Journal of Econometrics 105.1 (2001): 131-159.

Evans, M., \& Lyons, R. K. (2002). Order Flow and Exchange Rate Dynamics. Journal of Political Economy, 110(1), 170-180.

Gonzalo, J. and J-Y Pitarakis (2012). "Regime specific predictability in predictive regressions." Journal of Business and Economic Statistics, 30(2), 229-241.

Hall, P. and C.C. Heyde (1980). Martingale Limit Theory and its Application. Academic Press.

Homm, U., and Breitung, J., (2012), Testing for speculative bubbles in stock markets: a comparison of alternative methods. Journal of Financial Econometrics, 10(1):198-231.

Jansson, M and M. Moreira (2006). "Optimal inference in regression models with nearly integrated regressors," Econometrica, 74(3), 681-715.

Kasparis, I., P. C. B. Phillips and T. Magdalinos, (2014). "Nonlinearity Induced Weak Instrumentation", Econometric Reviews, 33, 676-712.

Kostakis, A., T. Magdalinos, and M. P. Stamatogiannis, (2014). "Robust econometric inference for stock return predictability." Review of Financial Studies, hhu139. 
Lee, J.H. (2014). "Predictive quantile regression with persistent covariates: IVX-QR Approach," forthcoming in Journal of Econometrics.

Lee, J.H. (2014b). Online Supplement to "Predictive Quantile Regression with Persistent Covariates: IVX-QR Approach". Available at https://sites.google.com/site/jihyung412/research.

Lee, J.H. (2015). "Martingale Decomposition and Approximation for Nonlinearly Dependent Processes," Unpublished Manuscript: https://sites.google.com/site/jihyung412/research.

Lütkepohl, H. (2005). New Introduction to Multiple Time Series Analysis. New York: Springer.

Magdalinos, T. and P. C. B Phillips (2009a). "Limit theory for cointegrated systems with moderately integrated and moderately explosive regressors". Econometric Theory, 25, 482-526.

Magdalinos, T. and P. C. B Phillips (2009b). "Econometric inference in the vicinity of unity". CoFie Working Paper (7), Singapore Management University.

Mikusheva, A. (2007). Uniform inference in autoregressive models. Econometrica, 75(5), 14111452.

Miller, J. I., Park, J. Y. (2010). Nonlinearity, nonstationarity, and thick tails: How they interact to generate persistence in memory. Journal of Econometrics, 155:83-89.

Phillips, P. C. B. (1987). "Towards a unified asymptotic theory for autoregression," Biometrika $74,535-547$.

Phillips, P. C. B. (2007). "Unit root periodogram regression," Journal of Econometrics 138, $104-124$.

Phillips, P. C. B. and J.H. Lee (2015a). "Asset Pricing with Financial Bubble Risk," Unpublished Manuscript.

Phillips, P. C. B. and J.H. Lee (2015b). "Limit Theory VARs with Mixed Roots Near Unity," Econometric Reviews, 34, pp.1035-1056.

Phillips, P. C. B. and J.H. Lee (2015c). Online Supplement to: "Robust Econometric Inference with Mixed Integrated and Mildly Explosive Regressors", https://sites.google.com/site/jihyung412/research.

Phillips, P. C. B, and J.H. Lee (2013). "Predictive regression under various degrees of persistence and robust long-horizon regression". Journal of Econometrics, 177(2), 250-264.

Phillips, P. C. B., T. Magdalinos (2007a), "Limit Theory for Moderate Deviations from a Unit Root," Journal of Econometrics 136, 115-130.

Phillips, P. C. B., T. Magdalinos (2007b), "Limit Theory for Moderate Deviations from a Unit Root Under Weak Dependence," in G. D. A. Phillips and E. Tzavalis (Eds.) The Refinement of Econometric Estimation and Test Procedures: Finite Sample and Asymptotic Analysis. Cambridge: Cambridge University Press, pp.123-162. 
Phillips, P. C. B., and T. Magdalinos (2009), "Unit Root and Cointegrating Limit Theory when Initialization is in the Infinite Past", Econometric Theory, 25, 1682-1715.

Phillips, P. C. B., T. Magdalinos (2013), "Inconsistent VAR Regression with Common Explosive Roots," Econometric Theory, 29(04), 808-837.

Phillips, P.C.B., S. Shi, and Y. Yu (2015a). "Testing for Multiple Bubbles: Historical episodes of exuberance and collapse in the S\&P 500 ", International Economic Review, 2015 (forthcoming).

Phillips, P.C.B., S. Shi, and Y. Yu (2015b). "Testing for Multiple Bubbles: Limit theory of Real Time Detectors", International Economic Review, (forthcoming).

Phillips, P. C. B. and V. Solo (1992). "Asymptotics for Linear Processes," Annals of Statistics 20, 971-1001.

Phillips P. C. B., Y. Wu and J. Yu (2011). "Explosive behavior in the 1990s Nasdaq: When did exuberance escalate asset values?", International Economic Review, 52, pp. 201-226.

Phillips P. C. B. and J. Yu (2011). "Dating the Timeline of Financial Bubbles during the Subprime Crisis," Quantitative Economics, 2, pp. 455-491.

Rossi, Barbara. "Exchange Rate Predictability." Journal of Economic Literature 51.4 (2013): 1063-1119.

Sager, M., \& Taylor, M. P. (2008). Commercially available order flow data and exchange rate movements: Caveat emptor. Journal of Money, Credit and Banking, 40(4), 583-625.

Stock, J. (1991). "Confidence intervals for the largest autoregressive root in US macroeconomic time series," Journal of Monetary Economics, 28(3), 435-459.

Wu, W. B. (2007). Strong invariance principles for dependent random variables. The Annals of Probability, 35(6), 2294-2320.

Wu, W. B. (2011). Asymptotic theory for stationary processes. Statistics and Its Interface, 0, 1-20. 